\title{
RELATIVE HOMOLOGICAL ALGEBRA FOR THE PROPER CLASS $\omega_{f}$
}

\author{
GRIGORY GARKUSHA
}

The classes of $f p$-injective and $f p$-flat modules have been introduced in [8] to study $F P$-injective and weakly quasi-Frobenius rings. Both classes are definable (=elementary) in the first order language of modules and naturally generalize the corresponding classes of $F P$-injective and flat modules.

Homological algebra based on FP-injective and flat modules is well studied and was developed in the 70 -s (see e.g. $[\mathbf{1 4}, \mathbf{2 3}, \mathbf{2 4}]$ ). One would like to construct homological algebra based on $f p$-injective and $f p$-flat modules. In this paper we shall show that such homological algebra can be realized as relative homological algebra corresponding to the proper class (in the sense of [11]) of monomorphisms

$$
\omega_{f}=\left\{\mu \mid R^{I} \otimes \mu \text { is a monomorphism for any power } R^{I} \text { of a ring } R\right\} .
$$

If we define the corresponding relative injective and relative flat modules in the natural way, we call them $f$-injective and $f$-flat modules respectively, then they will correspond to $f p$-injective pure-injective modules and $f p$-flat modules.

There are many results of a homological nature which may be generalized from Noetherian rings to coherent rings. In this direction, finitely generated modules are replaced by finitely presented modules and injective modules should be replaced by $F P$-injective modules (see $[\mathbf{2 3}, \mathbf{2 4}]$ ). In our situation analogous results, which are related to the relative homological algebra we construct in this paper, are valid for all rings. This phenomenon can be explained as follows. The classical Chase theorem (see [25]) states that a ring $R$ is left coherent iff each power $R^{I}$ of $I$ copies of the ring $R$ is a flat right $R$-module. But if we consider only those exact sequences under which the functor $R^{I} \otimes_{R}$ - is exact, we shall arrive at the proper class $\omega_{f}$.

Every time we deal with definable subcategories the category of generalized modules

$$
{ }_{R} \mathcal{C}=(\bmod R, \mathrm{Ab})
$$

is of great utility in this context as well as torsion theories of finite type of ${ }_{R} \mathcal{C}$ (consult e.g. the works $[\mathbf{1 3}, \mathbf{1 8}])$. For this reason the majority of statements of the paper is proved by using the technique of torsion/localizing functors in ${ }_{R} \mathrm{e}$.

The paper is organized as follows. The first two sections are preliminary. There we present the necessary category-theoretic background and introduce the proper class $\omega_{f}$ as a class of monomorphisms of a certain quotient category in ${ }_{R} \mathcal{C}$. In section 3 the classes of $f$-injective and $f$-flat modules are investigated. In section 4 we study basic properties for the functors $\operatorname{Ext}_{f}$ and $\operatorname{Tor}^{f}$ which are related to $f$-injective and $f$-flat resolutions. Also, certain homological dimensions are discussed in this section. In section 5 we describe the relative derived category $D_{f}(R)$ and the functor Ext $f$ as Hom-sets in $D_{f}(R)$. In the remaining

2000 Mathematics Subject Classification. 18G25, 16E10, 16E30. 
section we study the relationship of the $G$-theory of a ring with the $K$-theory of some abelian subcategories of ${ }_{R} \mathcal{C}$.

Throughout the paper $R$ denotes a ring with identity. The category of left (respectively right) $R$-modules is denoted by $R \operatorname{Mod}($ respectively $\operatorname{Mod} R$ ) and the category of finitely presented left (respectively right) $R$-modules by $R \bmod (\operatorname{respectively~} \bmod R$ ).

\section{The CATEgory of Generalized Modules}

\section{The category of generalized left $R$-modules}

$$
{ }_{R} \mathrm{C}=(\bmod R, \mathrm{Ab})
$$

consists of additive covariant functors defined on the category of finitely presented right $R$ $\operatorname{modules} \bmod R$ with values in the category of abelian groups $\mathrm{Ab}$. In this section we collect basic facts about the category ${ }_{R} \mathcal{C}$. For details and proofs we refer the reader to $[\mathbf{1 3}, \mathbf{1 8}]$, for example. All subcategories considered are assumed to be full.

We say that a subcategory $\mathcal{S}$ of an abelian category $\mathcal{C}$ is a Serre subcategory if for any short exact sequence

$$
0 \longrightarrow X \longrightarrow Y \longrightarrow Z \longrightarrow 0
$$

in $\mathcal{C}$ an object $Y \in \mathcal{S}$ if and only if $X, Z \in \mathcal{S}$. A Serre subcategory $\mathcal{S}$ of a Grothendieck category $\mathcal{C}$ is localizing if it is closed under taking direct limits. Equivalently, the inclusion functor $i: \mathcal{S} \rightarrow \mathcal{C}$ admits the right adjoint $t=t_{\mathcal{S}}: \mathcal{C} \rightarrow \mathcal{S}$ which takes every object $X \in \mathcal{C}$ to the maximal subobject $t(X)$ of $X$ belonging to $\mathcal{S}$ [16]. The functor $t$ we call the torsion functor. An object $C$ of ${ }_{R} \mathcal{C}$ is said to be $\mathcal{S}$-torsionfree if $t(C)=0$. Given a localizing subcategory $\mathcal{S}$ of ${ }_{R} \mathcal{C}$ the quotient category ${ }_{R} \mathrm{C} / \mathcal{S}$ consist of $C \in{ }_{R} \mathcal{C}$ such that $t(C)=t^{1}(C)=0$. The objects from ${ }_{R} \mathcal{C} / \mathcal{S}$ we call $\mathcal{S}$-closed objects. Given $C \in{ }_{R} \mathcal{C}$ there exists a canonical exact sequence

$$
0 \longrightarrow A^{\prime} \longrightarrow C \stackrel{\lambda_{C}}{\longrightarrow} C_{\mathcal{S}} \longrightarrow A^{\prime \prime} \longrightarrow 0
$$

with $A^{\prime}=t(C), A^{\prime \prime} \in \mathcal{S}$, and where $C_{\mathcal{S}} \in{ }_{R} \mathcal{C} / \mathcal{S}$ is the maximal essential extension of $\widetilde{C}=C / t(C)$ such that $C_{\mathcal{S}} / \widetilde{C} \in \mathcal{S}$. The object $C_{\mathcal{S}}$ is uniquely defined and is called the $\mathcal{S}$-envelope of $C$. Moreover, the inclusion functor $i:{ }_{R} \mathcal{C} / \mathcal{S} \rightarrow{ }_{R} \mathcal{C}$ has the left adjoint localizing functor $(-)_{\mathcal{S}}:{ }_{R} \mathrm{C} \rightarrow{ }_{R} \mathrm{C} / \mathcal{S}$, which is also exact. It takes each $C \in{ }_{R} \mathrm{C}$ to $C_{\mathcal{S}} \in{ }_{R} \mathrm{e} / \mathcal{S}$. Then,

$$
\operatorname{Hom}_{R} \mathrm{e}(X, Y) \simeq \operatorname{Hom}_{R} \mathrm{e} / \mathcal{S}\left(X_{\mathcal{S}}, Y\right)
$$

for all $X \in{ }_{R} \mathcal{C}$ and $Y \in{ }_{R} \mathcal{C} / \mathcal{S}$.

An object $X$ of a Grothendieck category $\mathcal{C}$ is finitely generated if whenever there are subobjects $X_{i} \subseteq X$ with $i \in I$ satisfying $X=\sum_{i \in I} X_{i}$, then there is a finite subset $J \subset I$ such that $X=\sum_{i \in J} X_{i}$. The subcategory of finitely generated objects is denoted by fg $\mathcal{C}$. A finitely generated object $X$ is said to be finitely presented if every epimorphism $\gamma: Y \rightarrow X$ with $Y \in$ fg $\mathcal{C}$ has the finitely generated kernel Ker $\gamma$. By fp $\mathcal{C}$ we denote the subcategory consisting of finitely presented objects. Finally, we refer to a finitely presented object $X \in \mathcal{C}$ as coherent if every finitely generated subobject of $X$ is finitely presented. The corresponding subcategory of coherent objects will be denoted by coh $\mathcal{C}$.

The category ${ }_{R} \mathcal{C}$ is a locally coherent Grothendieck category, i.e. every object $C \in{ }_{R} \mathcal{C}$ is a direct limit $C=\underline{\lim }_{I} C_{i}$ of coherent objects $C_{i} \in \operatorname{coh}_{R} \mathcal{C}$. Equivalently, the category $\operatorname{coh}_{R} \mathcal{C}$ is 
abelian. Moreover, ${ }_{R} \mathcal{C}$ has enough coherent projective generators $\{(M,-)\}_{M \in \bmod R}$. Thus, every coherent object $C \in \operatorname{coh}_{R} \mathcal{C}$ has a projective presentation

$$
(N,-) \longrightarrow(M,-) \longrightarrow C \longrightarrow 0,
$$

where $M, N \in \bmod R$.

A localizing subcategory $\mathcal{S}$ of ${ }_{R} \mathrm{C}$ is of finite type if the inclusion functor $i:{ }_{R} \mathrm{C} / \mathcal{S} \rightarrow{ }_{R} \mathrm{C}$ commutes with direct limits. Then the quotient category ${ }_{R} \mathrm{C} / \mathcal{S}$ is locally coherent and $\operatorname{coh}_{R} \mathrm{C} / \mathcal{S}=\left\{C_{\mathcal{S}} \mid C \in \operatorname{coh}_{R} \mathcal{C}\right\}$.

Proposition 1.1. For a localizing subcategory $\mathcal{S}$ of ${ }_{R} \mathcal{C}$ the following are equivalent:

(1) $\mathcal{S}$ is of finite type;

(2) the inclusion functor $i:{ }_{R} \mathrm{C} / \mathcal{S} \rightarrow{ }_{R} \mathrm{C}$ commutes with direct unions;

(3) the torsion functor ts commutes with direct limits.

Proof. The equivalence $(1) \Leftrightarrow(2)$ is a consequence of [6, Theorem 5.14]; (1) $\Leftrightarrow(3)$ follows from [16, Lemma 2.4].

By a result of Herzog and Krause $[\mathbf{1 3}, \mathbf{1 6}]$ a localizing subcategory in ${ }_{R} \mathcal{C}$ is of finite type iff it has the form $\overrightarrow{\mathcal{S}}$ with $\mathcal{S}$ some Serre subcategory of $\operatorname{coh}_{R} \mathcal{C}$ and

$$
\overrightarrow{\mathcal{S}}=\left\{\lim _{i \in I} C_{i} \mid C_{i} \in \mathcal{S}\right\} .
$$

We say that $M \in{ }_{R} \mathcal{C}$ is a coh-injective object if $\operatorname{Ext}_{R}^{1} \mathrm{e}(C, M)=0$ for any $C \in \operatorname{coh}_{R} \mathcal{C}$. The fully faithful functor $-\otimes_{R}$ ? $: R \operatorname{Mod} \rightarrow{ }_{R} \mathrm{C}, M \mapsto-\otimes_{R} M$, identifies $R \operatorname{Mod}$ with the subcategory of coh-injective objects of ${ }_{R} \mathcal{C}$. Moreover, the functor $-\otimes_{R} M \in \operatorname{coh}_{R} \mathcal{C}$ iff $M \in R \bmod$. Given $C \in \operatorname{coh}_{R} \mathcal{C}$ there is an exact sequence

$$
0 \longrightarrow C \longrightarrow-\otimes_{R} M \longrightarrow-\otimes_{R} N
$$

in $\operatorname{coh}_{R} \mathrm{e}$ with $M, N \in R$ mod.

A monomorphism $\mu: M \rightarrow N$ in $R$ Mod is a pure monomorphim if for any $K \in$ $\operatorname{Mod} R$ the morphism $K \otimes \mu$ is a monomorphism. Equivalently, the ${ }_{R} \mathcal{C}$-morphism $-\otimes \mu$ is a monomorphism. A module $Q$ is pure-injective if the functor $\operatorname{Hom}_{R}(-, Q)$ takes the pure monomorphisms to epimorphisms. The injective objects of ${ }_{R} \mathcal{C}$ are precisely the objects of the form $-\otimes_{R} Q$ with $Q$ a pure-injective module.

Let $M$ be a left module; then

$$
\mathcal{S}_{M}=\left\{C \in \operatorname{coh}_{R} \mathcal{C} \mid\left(C,-\otimes_{R} M\right)=0\right\}
$$

is Serre. Furthermore, every Serre subcategory of $\operatorname{coh}_{R} \mathcal{C}$ arises in this fashion.

Proposition 1.2. Let $\mathcal{S}_{M}$ be the Serre subcategory of $\mathrm{coh}_{R} \mathcal{C}$ cogenerated by a left $R$-module $M$. Denote by $X$ the subcategory of $R$ Mod consisting of the modules of the form $\lim _{k \in K} M^{I_{k}}$ with some sets of indices $K$ and $I_{k}, k \in K$, and $M^{I_{k}}$ the product of $I_{k}$ copies of $M$. Then an object $F$ of ${ }_{R} \mathcal{C}$ is $\mathcal{S}_{M}$-torsionfree iff it is a subobject of $-\otimes_{R} L$ with $L \in \mathcal{X}$. Moreover,

$$
\overrightarrow{\mathcal{S}}_{M}=\left\{F \in{ }_{R} \mathcal{Q} \mid\left(F,-\otimes_{R} L\right)=0 \text { for all } L \in X\right\} .
$$

Proof. The proof is like that of [18, Theorem 2.1]. Let $C \in \operatorname{coh}_{R} \mathcal{C}$ and $I_{C}$ denote the set $\left(C,-\otimes_{R} M\right)$. We have an exact sequence

$$
0 \longrightarrow X \longrightarrow C \stackrel{\rho}{\longrightarrow}-\otimes_{R} M^{I_{C}} .
$$


Here we use the relation $\left(-\otimes_{R} M\right)^{I_{C}}=-\otimes_{R} M^{I_{C}}$. We claim that $X=t(C)$. Indeed, if we apply the left exact $\mathcal{S}_{M}$-torsion functor to (1.1), we shall obtain

$$
0 \longrightarrow t(X) \longrightarrow t(C) \longrightarrow t\left(-\otimes_{R} M^{I_{C}}\right)=0 .
$$

Therefore $t(C)=t(X) \subseteq X$. Let $Y$ be a finitely generated subobject of $X$ such that there exists a non-zero morphism $\varphi: Y \rightarrow-\otimes_{R} M$. Since $-\otimes_{R} M$ is coh-injective, $\varphi$ can be extended to a non-zero morphism $\psi: C \rightarrow-\otimes_{R} M$. But $\psi$ factors through $\rho$. Hence $\varphi=0$, a contrudiction to our assumption. Thus $X=t(C)$.

Consider a direct limit of coherent objects $C=\underline{\lim }_{k \in K} C_{k}$. Then the sequence

$$
0 \longrightarrow \lim _{\longrightarrow} t\left(C_{k}\right)=t(C) \longrightarrow C \longrightarrow \lim _{k} C_{k} / t\left(C_{k}\right)=C / t(C) \longrightarrow 0
$$

is exact. Given $k \in K$ let $J_{k}=\{j \in K \mid k \leqslant j\}$ and $I_{k}=I_{C_{k}} \times J_{k}$. The canonically defined monomorphisms $C_{k} / t\left(C_{k}\right) \rightarrow-\otimes_{R} M^{I_{k}}, k \in K$, induce a monomorphism $C / t(C) \rightarrow$ $-\otimes_{R} L$ with $L=\lim _{k \in K} M^{I_{k}} \in \mathcal{X}$. Thus the $\mathcal{S}_{M}$-torsionfree objects of ${ }_{R} \mathcal{C}$ are precisely the subobjects of the functors $-\otimes_{R} L$ with $L \in X$.

Next, it is easy to see that $\left(C,-\otimes_{R} L\right)=0$ for $C \in \overrightarrow{\mathcal{S}}_{M}$ and $L \in X$. Given an object $F \in$ ${ }_{R} \mathcal{C}$ the relation $\left(F,-\otimes_{R} L\right)=0$, for all $L \in \mathcal{X}$, implies the relation $\left(F / t(F),-\otimes_{R} L\right)=0$. Therefore $F=t(F)$, as claimed.

We refer to the subcategory

$$
z=\left\{N \in R \operatorname{Mod} \mid-\otimes_{R} N \text { is } \mathcal{S}_{M} \text {-torsionfree }\right\}
$$

as a definable subcategory.

Corollary 1.3. A left $R$-module belongs to $Z$ iff it is a pure submodule of a module $L$ from $x$.

In the sequel, we use the following Serre subcategories of $\operatorname{coh}_{R} \mathrm{C}$ :

$$
\begin{gathered}
\mathcal{S}^{R}=\left\{C \in \operatorname{coh}_{R} \mathcal{C} \mid C(R)=0\right\} \\
\mathcal{S}_{R}=\left\{C \in \operatorname{coh}_{R} \mathcal{C} \mid\left(C,-\otimes_{R} R\right)=0\right\},
\end{gathered}
$$

as well as the localizing subcategories of finite type $\overrightarrow{\mathcal{S}}^{R}$ and $\overrightarrow{\mathcal{S}}_{R}$

$$
\begin{aligned}
& \overrightarrow{\mathcal{S}}^{R}=\left\{C \in{ }_{R} \mathcal{C} \mid C=\underline{\left.\lim _{\longrightarrow} C_{i}, C_{i} \in \mathcal{S}^{R}\right\}}\right. \\
& \overrightarrow{\mathcal{S}}_{R}=\left\{C \in{ }_{R} \mathcal{C} \mid C=\underline{\lim _{\longrightarrow}} C_{i}, C_{i} \in \mathcal{S}_{R}\right\} .
\end{aligned}
$$

The corresponding $\overrightarrow{\mathcal{S}}^{R}$-torsion and $\overrightarrow{\mathcal{S}}_{R^{\text {-torsion }}}$ functors will be denoted by $t_{\mathcal{S}^{R}}$ and $t_{\mathcal{S}_{R}}$ respectively.

\section{Proper Classes}

Let $T$ denote the functor that takes a left $R$-module $M$ to the object $\left(-\otimes_{R} M\right)_{\mathcal{S}^{R}}$ in ${ }_{R} \mathrm{C} / \overrightarrow{\mathcal{S}}^{R}$.

Lemma 2.1. The functor $T$ is fully faithful, right exact and preserves direct limits.

Proof. Let $\mathcal{P}^{R}$ denote the localizing subcategory $\left\{F \in{ }_{R} \mathcal{C} \mid F(R)=0\right\}$ in ${ }_{R} \mathcal{C}$. Then the functor $M \mapsto P(M)=\left(-\otimes_{R} M\right)_{\mathcal{P}^{R}}$ induces an equivalence between $R \operatorname{Mod}$ and ${ }_{R} \mathrm{C} / \mathcal{P}^{R}[\mathbf{9}]$. A quasi-inverse functor to $P$ is induced by the functor ${ }_{R} \mathrm{C} \rightarrow \operatorname{Mod} R$ taking an object $F$ of ${ }_{R} \mathcal{C}$ to $F(R)$. Since $\overrightarrow{\mathcal{S}}^{R} \subseteq \mathcal{P}^{R}$ the functor $P$ factors as $P=L \circ T$, where $L:{ }_{R} \mathcal{C} / \overrightarrow{\mathcal{S}}^{R} \rightarrow{ }_{R} \mathcal{C} / \mathcal{P}^{R}$ 
is the localization functor with respect to the localizing subcategory $\mathcal{P}^{R} / \overrightarrow{\mathcal{S}}^{R}$. It follows that $T$ is faithful.

To show that $T$ is full, it suffices to prove that the map $L(\mu): L T(M) \rightarrow L T(N)$ with $0 \neq \mu: T(M) \rightarrow T(N)$ is non-zero. Assume the converse. Then $\mu(R)=0$ and, hence, $D=$ Coker $\mu$ belongs to $\mathcal{P}^{R}$.

Let $\lambda: T(N) \rightarrow L T(N)$ denote the $\mathcal{P}^{R}$-envelope of $T(N)$ in ${ }_{R} \mathcal{C}$. Then $\lambda \mu=0$ and therefore $\lambda$ factors through $D$. Since $L T(N)$ is $\mathcal{P}^{R}$-torsionfree, wee see that $\lambda=0$. This implies that $T(N)$ belongs to $\mathcal{P}^{R}$ and thus $N=0$. It follows that $T(N)=0$, a contradiction.

Since both the tensor functor and the localizing functor preserve direct limits (right exact), we see that $T$ preserves direct limits (is right exact) as well.

A class of monomorphisms $\omega$ of $R$ Mod is proper if it satisfies the following axioms:

$P 0$. $\omega$ contains all split monomorphisms;

$P 1$. the composition of two monomorphisms in $\omega$, if defined, is also in $\omega$;

$P 2$. if a pushout diagram

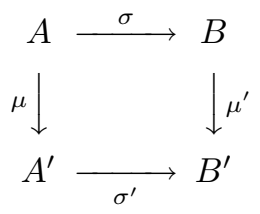

has $\sigma \in \omega$, then $\sigma^{\prime} \in \omega$;

P3. if $\sigma \tau \in \omega$, then $\tau \in \omega$.

The axioms $P 0^{\prime}-P 3^{\prime}$ for a proper class of epimorphisms are dually defined.

Proposition 2.2. The class of $R$-monomorphisms

$$
\omega_{f}=\{\mu \mid T \mu \text { is a monomorphism }\}
$$

satisfies the axioms $P 0-P 3$.

Proof. The axioms $P 0-P 1$ and $P 3$ are obvious. If the square (2.1) is pushout, then the sequence

$$
A \stackrel{(-\mu, \sigma)^{T}}{\longrightarrow} A^{\prime} \bigoplus B \stackrel{\left(\sigma^{\prime}, \mu^{\prime}\right)}{\longrightarrow} B^{\prime} \longrightarrow 0
$$

with $\left(\sigma^{\prime}, \mu^{\prime}\right)=\operatorname{coker}(-\mu, \sigma)^{T}$ is exact. By Lemma 2.1 the sequence

$$
T A \stackrel{(-T \mu, T \sigma)^{T}}{\longrightarrow} T A^{\prime} \bigoplus T B \stackrel{\left(T \sigma^{\prime}, T \mu^{\prime}\right)}{\longrightarrow} T B^{\prime} \longrightarrow 0
$$

is exact. Thus $T B^{\prime}=T A^{\prime} \coprod_{T A} T B$. Since $T \sigma$ is a monomorphism, it follows that $T \sigma^{\prime}$ is a monomorphism. This implies the claim.

By Lemma 2.1 the proper class $\omega_{f}$ is inductively closed, i.e. given any direct system $\left\{\mu_{i}\right\}_{i \in I}$ of monomorphisms from $\omega_{f}$ the morphism $\underline{\lim } \mu_{i}$ is in $\omega_{f}$. Below we shall show that

$$
\omega_{f}=\left\{\mu \mid R^{I} \otimes \mu \text { is a monomorphism for any power } R^{I} \text { of } R\right\} .
$$

The most obvious example of a monomorphism in $\omega_{f}$ is a pure monomorphism, because it is a direct limit of split monomorphisms [22, Theorem 6.2]. We say that a module $M$ is coinjective if any extension of $M$ belongs to $\omega_{f}$. As an example, every extension $\mu: M \rightarrow E$ of a finitely presented module $M \in R \bmod$ belongs to $\omega_{f}$. Indeed, if $X$ is a finitely generated 
subobject of $\operatorname{Ker}(-\otimes \mu)$, it is coherent and $X(R)=0$. Hence $\operatorname{Ker}(-\otimes \mu) \in \overrightarrow{\mathcal{S}}^{R}$. Therefore the morphism $T \mu$ is a monomorphism, i.e. $\mu \in \omega_{f}$.

Proposition 2.3. A ring $R$ is left coherent iff every left $R$-module is coinjective.

Proof. Let $R$ be left coherent. Then any monomorphism $\mu: M \rightarrow N$ is a direct limit $\mu=\lim _{I} \mu_{i}$ of monomorphisms $\mu_{i}$ in $R \bmod \left[\mathbf{1 7}\right.$, Lemma 5.9]. Since each $\mu_{i} \in \omega_{f}$, it follows that $\mu=\lim _{\longrightarrow} \mu_{i}$ is also in $\omega_{f}$. Therefore $M$ is coinjective.

Assume the converse. Since $\mu \in \omega_{f}$ iff $\operatorname{Ker}(-\otimes \mu) \in \overrightarrow{\mathcal{S}}^{R}$ and since any monomorphism belongs to $\omega_{f}$, our assertion follows from $[\mathbf{9}$, Theorem 2.5].

A module $M \in R$ Mod is a $f$-submodule of $N \in R \operatorname{Mod}$ (respectively $f$-quotient module) if there is a monomorphism $\mu: M \rightarrow N$ with $\mu \in \omega_{f}$ (respectively if there is an epimorphism $\mu: N \rightarrow M$ with $\left.\operatorname{ker} \mu \in \omega_{f}\right)$. A short exact sequence

$$
0 \longrightarrow M \longrightarrow N \longrightarrow L \longrightarrow 0
$$

is $f$-exact if $M$ is a $f$-submodule of $N$. Equivalently, if $L$ is a $f$-quotient module of $N$. Clearly, the exact sequence $(2.2)$ is $f$-exact iff the sequence

$$
0 \longrightarrow T M \longrightarrow T N \longrightarrow T L \longrightarrow 0
$$

is exact in ${ }_{R} \mathcal{C} / \overrightarrow{\mathcal{S}}^{R}$.

We refer to a morphism $\mu: M \rightarrow N$ as an $f$-epimorphism if $N$ is a $f$-quotient of $M$. The class of $f$-epimorphisms will be denoted by $\omega^{f}$. A morphism $\mu: M \rightarrow N$ is an $f$-homomorphism if $\mu=\sigma \tau$ with $\sigma \in \omega_{f}$ and $\tau \in \omega^{f}$.

\section{3. $f$-injeCtive AND $f$-FLAT MOdULES}

In this section we study relative injective and relative flat modules for the proper class $\omega_{f}$.

Definitions. (1) A left $R$-module $M$ is said to be $F P$-injective (or absolutely pure) if every monomorphism $\mu: M \rightarrow N$ is pure. Equivalently, for all $F \in R \bmod$ we have: $\operatorname{Ext}_{R}^{1}(F, M)=0[\mathbf{2 4}]$. A ring $R$ is left $F P$-injective if the module ${ }_{R} R$ is $F P$-injective.

(2) $M$ is an $f p$-injective module if for every monomorphism $\mu: K \rightarrow L$ in $R$ mod the morphism $(\mu, M)$ is an epimorphism. Clearly, $F P$-injective modules are $f p$-injective and every finitely presented $f p$-injective module is $F P$-injective.

(3) $M$ is said to be $f p$-flat if for every monomorphism $\mu: K \rightarrow L$ in $\bmod R$ the morphism $\mu \otimes M$ is a monomorphism. Clearly, every flat left $R$-module is $f p$-flat. The converse holds iff the ring $R$ is right coherent [8, Theorem 2.4].

Let the functor $S$ take a module $M \in R$ Mod to the functor $S M=\left(-\otimes_{R} M\right)_{\mathcal{S}_{R}}$.

Proposition 3.1. The following statements are true:

(1) a module $M$ is fp-injective iff $-\otimes_{R} M=T M$;

(2) a module $M$ is fp-flat iff $-\otimes_{R} M=S M$;

(3) the subcategory of $f p$-injective (fp-flat) modules is definible.

Moreover, every object TM (SM) with $M$ fp-injective ( $f p$-flat) is coh-injective in ${ }_{R} \mathcal{C} / \overrightarrow{\mathcal{S}}^{R}$ $\left({ }_{R} \mathrm{e} / \overrightarrow{\mathcal{S}}_{R}\right)$. 
Proof. By [8, Proposition 2.2] $M$ is $f p$-injective ( $f p$-flat) iff $-\otimes_{R} M$ is $\mathcal{S}^{R}$-torsionfree $\left(\mathcal{S}_{R^{-}}\right.$ torsionfree). This implies (3). The rest of the proof is a consequence of [13, Proposition 3.10].

The Auslander-Gruson-Jensen duality $D$ (see $[\mathbf{1 , 1 2}])$ takes each object $C \in \operatorname{coh}_{R} \mathrm{C}$ to the object $D C \in \operatorname{coh} \mathcal{C}_{R}$ defined as follows:

$$
(D C)\left({ }_{R} N\right)=\operatorname{Hom}_{R} \mathrm{e}\left(C,-\otimes_{R} N\right)
$$

and a morphism $\alpha$ of $\operatorname{coh}_{R} \mathcal{C}$ to the morphism $D \alpha$ of $\operatorname{coh} \mathcal{C}_{R}$ defined by the rule:

$$
(D \alpha)\left({ }_{R} N\right)=\operatorname{Hom}_{R} \mathrm{e}\left(\alpha,-\otimes_{R} N\right) .
$$

Proposition 3.2. [29, Lemma 2], [13, Proposition 5.6] Let ${ }_{S} M_{R}$ be an $(S, R)$-bimodule and let ${ }_{S} E$ be an injective $S$-module. Then for each $C \in \operatorname{coh}_{R}$ there is an isomorphism

$$
\operatorname{Hom}_{S}\left({ }_{S}\left(C, M \otimes_{R}-\right),{ }_{S} E\right) \simeq \operatorname{Hom}_{R} \mathrm{e}\left(D C,-\otimes_{R}\left({ }_{S} M_{R},{ }_{S} E\right)\right)
$$

natural in $C$.

Throughout the paper the character module $\operatorname{Hom}_{\mathbf{Z}}(M, \mathbf{Q} / \mathbf{Z})$ of a module $M$ is denoted by $\widehat{M}$.

Since

$$
D \mathcal{S}^{R}={ }_{R} \mathcal{S}=\left\{C \in \operatorname{coh} \mathcal{C}_{R} \mid\left(C, R \otimes_{R}-\right)=0\right\}
$$

and

$$
D \mathcal{S}_{R}={ }^{R} \mathcal{S}=\left\{C \in \operatorname{coh}_{R} \mid C(R)=0\right\}
$$

it follows from [8, Proposition 2.2] the following.

Corollary 3.3. A left R-module $M$ is fp-injective (fp-flat) iff its character module $\widehat{M}$ is fp-flat (fp-injective).

We observe that the Serre subcategory $\mathcal{S}^{R}$ of $\operatorname{coh}_{R} \mathcal{C}$ is cogenerated by any injective cogenerator, say $\widehat{R}$, of $R$ Mod.

Proposition 3.4. A left $R$-module is fp-injective (fp-flat) iff it is a pure submodule of $\lim _{k \in K} \widehat{R}^{I_{k}}\left(\mathrm{lim}_{k \in K} R^{I_{k}}\right)$ for some sets of indices $K$ and $I_{k}, k \in K$.

Proof. This follows from Proposition 1.2 and Corollary 1.3.

A left $R$-module $Q$ is said to be $f$-injective (respectively $f$-projective) if the functor $\operatorname{Hom}_{R}(-, Q)$ (respectively $\operatorname{Hom}_{R}(Q,-)$ ) takes the monomorphisms of $\omega_{f}$ (respectively the epimorphisms of $\left.\omega^{f}\right)$ to epimorphisms.

Proposition 3.5. An R-module $Q$ is $f$-injective iff it is a fp-injective pure-injective module.

Proof. Since an injective object $-\otimes_{R} Q$ of ${ }_{R} \mathcal{C}$ is injective in ${ }_{R} \mathcal{C} / \overrightarrow{\mathcal{S}}^{R}$ iff it is $\mathcal{S}^{R}$-closed, Proposition 3.1 implies that the ${ }_{R} \mathcal{C} / \overrightarrow{\mathcal{S}}^{R}$-injective objects are precisely the $f p$-injective pureinjective modules.

Suppose that $M$ is $f$-injective; then the ${ }_{R} \mathcal{C} / \overrightarrow{\mathcal{S}}^{R}$-monomorphism $T M \rightarrow-\otimes_{R} Q=T Q$ with $-\otimes_{R} Q=E(T M)$ splits. Therefore, $M$ is a pure-injective $f p$-injective module. The converse is easy. 
A $f$-monomorphism $\mu: M \rightarrow E=E_{f}(M)$ with $E$ a $f$-injective module is called a $f$ injective envelope of $M$ if any endomorphism $\varphi$ of $E$ such that $\varphi \mu=\mu$ is an isomorphism.

Lemma 3.6. A f-monomorphism $\mu: N \rightarrow E$ is a f-injective envelope iff $T \mu: T N \rightarrow T E$ is an injective envelope of $T N$ in ${ }_{R} \mathrm{C} / \overrightarrow{\mathcal{S}}^{R}$. If $\mu^{\prime}: N \rightarrow E^{\prime}$ is another $f$-injective envelope, then there exists an isomorphism $\psi: E \rightarrow E^{\prime}$ such that $\psi \mu=\mu^{\prime}$.

Proof. Straightforward.

The existence of injective envelopes in ${ }_{R} \mathcal{C} / \overrightarrow{\mathcal{S}}^{R}$ implies the following.

Corollary 3.7. A f-injective envelope of a module always exists.

So we obtain that the proper class $\omega_{f}$ is injective, that is, every module is a $f$-submodule of a $f$-injective module. The projective proper classes are dually difined.

We refer to a right $R$-module $M$ as a $f$-flat module if the tensor functor $M \otimes_{R}-$ preserves $f$-exact sequences.

Theorem 3.8. A right $R$-module $M$ is $f$-flat iff it is fp-flat. Moreover, the class of $f$-flat $R$-modules is closed under taking products and direct limits.

Proof. Since every monomorphism $\mu$ in $R$ mod belongs to $\omega_{f}$, it follows that the $f$-flat $R$-modules are $f p$-flat $R$-modules.

Consider a $f p$-flat right $R$-module $M$ and a $f$-monomorphism $\mu: K \rightarrow L$. We want to show that $1 \otimes \mu: M \otimes_{R} K \rightarrow M \otimes_{R} L$ is a monomorphism. From [13, Proposition 4.3] it follows that the character module $\widehat{M}$ is pure-injective. On the other hand, $\widehat{M}$ is $f p$-injective by Corollary 3.3. Proposition 3.5 implies that $\widehat{M}$ is $f$-injective. We see that the map

$$
\operatorname{Hom}_{R}(L, \widehat{M}) \longrightarrow \operatorname{Hom}_{R}(K, \widehat{M})
$$

is an epimorphism. This map is isomorphic to the map

$$
\operatorname{Hom}_{\mathbf{Z}}\left(M \otimes_{R} L, \mathbf{Q} / \mathbf{Z}\right) \longrightarrow \operatorname{Hom}_{\mathbf{Z}}\left(M \otimes_{R} K, \mathbf{Q} / \mathbf{Z}\right) .
$$

Since $\mathbf{Q} / \mathbf{Z}$ is an injective cogenerator in $\mathrm{Ab}$, it follows that $1 \otimes \mu: M \otimes_{R} K \rightarrow M \otimes_{R} L$ is a monomorphism.

The fact that $f$-flat modules are closed under products and direct limits follows from $[\mathbf{8}$, Proposition 2.3].

Corollary 3.9. A module $M$ is fp-flat iff its character module $\widehat{M}$ is an fp-injective pureinjective module. In other words, a module is $f$-flat iff its character module is a f-injective module.

As for the class of flat $R$-modules by the Chase theorem [25, Proposition I.13.3] every product of flat right $R$-modules is flat iff the ring $R$ is left coherent.

The class $\omega_{f}$ is flatly generated by $f p$-flat modules, that is, $\mu \in \omega_{f}$ iff $M \otimes \mu$ is a monomorphism for any $f p$-flat module $M$. Indeed, if $\mu \in \omega_{f}$ and $M$ is $f p$-flat, Theorem 3.8 implies that $M \otimes \mu$ is a monomorphism.

On the other hand, if $M \otimes \mu$ with $\mu: K \rightarrow L$ is a monomorphism for each $f p$-flat module $M$, the map (3.1) is an epimorphism. In particular, it is an epimorphism for $M=\widehat{Q}$ with $Q$ a $f$-injective module and, hence, $\operatorname{Hom}_{R} \mathcal{e} / \overrightarrow{\mathcal{S}}^{R}\left(\operatorname{Ker} T \mu,-\otimes_{R} Q^{\uparrow \uparrow}\right)=0$. Since $Q$ is a 
pure submodule of $Q^{\Upsilon \Upsilon}$, it follows that $\operatorname{Hom}_{R} \mathcal{e} / \overrightarrow{\mathcal{s}}^{R}\left(\operatorname{Ker} T \mu,-\otimes_{R} Q\right)=0$. As $f$-injectives cogenerate ${ }_{R} \mathcal{C} / \overrightarrow{\mathcal{S}}^{R}$ this implies Ker $T \mu=0$, and, hence, $\mu \in \omega_{f}$.

Proposition 3.10. $\omega_{f}$ is flatly generated by modules $R^{I}$ with $I$ a set of indices, $R^{I}$ the product of I copies of $R$.

Proof. Let $F$ be a $f p$-flat module. By Proposition 3.4 it is a pure submodule of $\lim _{k \in K} R^{I_{k}}$ with $K, I_{k}, k \in K$, some sets of indices. Let $0 \rightarrow M \stackrel{\mu}{\rightarrow} N \rightarrow L \rightarrow 0$ be an exact sequence and the map $R^{I} \otimes \mu$ is a monomorphism for any set $I$. We have then the following commutative diagram with exact rows:

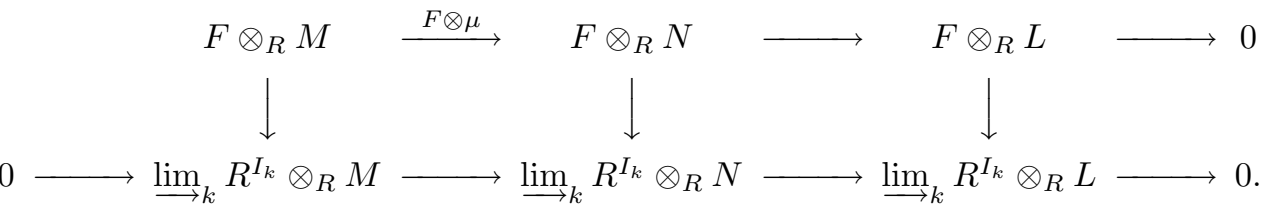

Since the vertical arrows are monomorphisms, $F \otimes \mu$ is a monomorphism. Thus $\mu \in \omega_{f}$, as claimed.

Below we discuss some properties of $f p$-injective and $f p$-flat modules we shall use later.

Proposition 3.11. For a left $R$-module $M$ the following statements are equivalent:

(1) $M$ is fp-injective;

(2) any $f$-exact sequence $0 \rightarrow M \rightarrow M^{\prime} \rightarrow M^{\prime \prime} \rightarrow 0$ is pure;

(3) there is a pure-exact sequence $0 \rightarrow M \rightarrow M^{\prime} \rightarrow M^{\prime \prime} \rightarrow 0$ with $M^{\prime}$ a fp-injective module;

(4) $\operatorname{Ext}_{R}^{1} \mathrm{e} / \overrightarrow{\mathcal{S}}^{R}(T F, T M)=0$ for all $F \in R$ mod.

Moreover, any product and any direct limit of fp-injective modules is also a fp-injective module.

Proof. (1) $\Longrightarrow(2)$. Let $F \in R \bmod$ then $T F \in \operatorname{coh}_{R} \mathcal{C} / \overrightarrow{\mathcal{S}}^{R}$. Since $T M$ is coh-injective in ${ }_{R} \mathrm{C} / \overrightarrow{\mathcal{S}}^{R}$ by Proposition 3.1, it follows that the sequence

$$
0 \longrightarrow(T F, T M) \longrightarrow\left(T F, T M^{\prime}\right) \longrightarrow\left(T F, T M^{\prime \prime}\right) \longrightarrow 0
$$

is exact. By Lemma 2.1 the sequence

$$
0 \longrightarrow(F, M) \longrightarrow\left(F, M^{\prime}\right) \longrightarrow\left(F, M^{\prime \prime}\right) \longrightarrow 0
$$

is exact. So, the sequence $0 \rightarrow M \rightarrow M^{\prime} \rightarrow M^{\prime \prime} \rightarrow 0$ is pure-exact.

$(2) \Longrightarrow(3)$. We may take $M^{\prime}$ to be a $f$-injective envelope of $M$.

$(3) \Longrightarrow(4)$. Since $-\otimes_{R} M$ is a subobject of the $\mathcal{S}^{R}$-torsionfree object $-\otimes_{R} M^{\prime}$, it is also $\mathcal{S}^{R}$-torsionfree. From [13, Proposition 3.10] it follows that $T M=-\otimes_{R} M$ is a coh-injective object for ${ }_{R} \mathcal{C} / \overrightarrow{\mathcal{S}}^{R}$.

(4) $\Longrightarrow(1)$. Let $\mu$ be a monomorphism in $R$ mod; then $T \mu$ is a monomorphism. By assumption, $(T \mu, T M)$ is an epimorphism. Since $T$ is full and faithful by Lemma 2.1 , the morphism $(\mu, M)$ is a monomorphism.

The fact that the class of $f p$-injective modules is closed under taking products and direct limits follows from [8, Proposition 2.3].

It is well-known that a direct limit of $F P$-injective left $R$-modules is $F P$-injective iff the ring $R$ is left coherent (see, e.g., $[\mathbf{2 4}]$ ). 
Corollary 3.12. If in a short $f$-exact sequence $0 \rightarrow M \rightarrow M^{\prime} \rightarrow M^{\prime \prime} \rightarrow 0$ modules $M$ and $M^{\prime}$ are $f p$-injective, then also $M^{\prime \prime}$ is fp-injective.

Proof. By the preceding proposition the sequence of the corollary is pure-exact. If we apply the right exact $\mathcal{S}^{R}$-torsion functor $t=t_{\mathcal{S}^{R}}$ to the ${ }_{R} \mathcal{C}$-exact sequence

$$
0 \longrightarrow-\otimes_{R} M \longrightarrow-\otimes_{R} M^{\prime} \longrightarrow-\otimes_{R} M^{\prime \prime} \longrightarrow 0
$$

we shall get an exact sequence

$$
0=t\left(-\otimes_{R} M^{\prime}\right) \longrightarrow t\left(-\otimes_{R} M^{\prime \prime}\right) \longrightarrow t^{1}\left(-\otimes_{R} M\right)=0 .
$$

Hence $M^{\prime \prime}$ is $f p$-injective by [8, Proposition 2.2].

The following two propositions extend the list of properties characterizing the coherent and Noetherian rings respectively (cf. $[\mathbf{2 3}]$ ).

Proposition 3.13. The following assertions are equivalent for a ring $R$ :

(1) $R$ is left coherent;

(2) the character module of a fp-injective left module is a flat module;

(3) a left module is fp-injective iff its character module is a flat module;

(4) a pure-injective envelope of a right $f$-flat module is a flat module.

Proof. (1) $\Longrightarrow(2)$. Over a left coherent ring every $f p$-flat right module is flat $[\mathbf{8}$, Theorem 2.4]. Now our implication follows from Corollary 3.3.

$(2) \Longrightarrow(3)$. This follows from Corollary 3.3.

$(3) \Longrightarrow(1)$. Suppose that the character module of an $f p$-injective left module $M$ is flat. Then $M$ is a pure submodule of the injective module $M^{\widehat{ }}$. Hence $M$ is a $F P$-injective module. Now our assertion follows from [8, Theorem 2.4].

$(1) \Longrightarrow(4)$. A pure-injective envelope $Q$ of a $f$-flat module $M$ is always a $f$-flat module. Indeed, the $\mathcal{C}_{R}$-injective functor $Q \otimes_{R}-$ is an injective invelope of $M \otimes_{R}-$ in $\mathcal{C}_{R}$. Since $M \otimes_{R}-$ is $\mathcal{S}_{R}$-torsionfree, it follows that $Q \otimes_{R}-$ is $\mathcal{S}_{R}$-torsionfree, too. Proposition 3.1 implies that $Q$ is $f$-flat. By assumption, $R$ is left coherent and therefore $Q$ is flat by $[\mathbf{8}$, Theorem 2.4].

$(4) \Longrightarrow(1)$. It is directly verified that given a pure-exact sequence

$$
0 \longrightarrow M \longrightarrow Q \longrightarrow N \longrightarrow 0
$$

with $Q$ flat, the module $M$ is also flat.

Suppose that a right $R$-module $M$ is $f$-flat. Then its pure-injective envelope $Q$ is flat by assumption. Hence $M$ is flat by the above. Therefore $R$ is left coherent by [8, Theorem 2.4].

Remark. By a recent result of Rothmaler [21] there are non-coherent rings over which pure-injective envelopes of flat modules are flat.

Proposition 3.14. The following assertions are equivalent for a ring $R$ :

(1) $R$ is left Noetherian;

(2) every left $f p$-injective module is an injective module;

(3) a left module is $f$-injective iff its character module is a flat module. 
Proof. (1) $\Longrightarrow(2)$. Since every left ideal $I$ of $R$ is finitely presented, then the representable functor $\operatorname{Hom}_{R}(-, M)$ takes the inclusion $I \subset R$ to an epimorphism whenever $M$ is $f p$ injective. Therefore $M$ is an injective module by the Baer criterion.

$(2) \Longrightarrow(1)$. It suffices to observe that every direct limit of $f p$-injective modules is a $f p$-injective module.

(1), (2) $\Longrightarrow(3)$. This implication follows from Proposition 3.13.

$(3) \Longrightarrow(2)$. Let $E$ be a $f$-injective envelope of a $f p$-injective module $M$. By Proposition $3.11 M$ is a pure submodule of $E$. Therefore $\widehat{M}$ is a direct summand of the flat module $\widehat{E}$. Then $M$ is $f$-injective by assumption. By the preceding proposition the ring $R$ is left coherent. Now our assertion follows from the fact that over the left coherent rings the classes of $f$-injective and injective left modules coincide (see [8, Theorem 2.4]).

We conclude the section by characterizing the class of $F P$-injective rings. We give the most interesting criteria for this class of rings in terms of $f$-flat and $f$-injective modules. For details and proofs we refer the reader to $[\mathbf{5}, \mathbf{8}]$.

We recall that $M \in R$ Mod is a $F P$-cogenerator if for any non-zero homomorphism $\rho: K \rightarrow L$ with $K$ a finitely generated $R$-module and $L$ a finitely presented module there exists a homomorphism $\mu: L \rightarrow M$ such that $\mu \rho \neq 0$. Equivalently, every finitely presented left $R$-module is a submodule of a product $M^{I}$ of some copies of the module $M$ [5].

Lemma 3.15. $M$ is a FP-cogenerator iff for any non-zero morphism $\rho: K \rightarrow L$ in $R \bmod$ there exists a morphism $\mu: L \rightarrow M$ such that $\mu \rho \neq 0$.

Proof. The necessary condition is trivial. Assume the converse. Let $\rho: K \rightarrow L$ be a non-zero homomorphism from a finitely generated $R$-module $K$ to a finitely presented module $L$. Then there exists an epimorphism $\pi: R^{n} \rightarrow K$. By assumption, there exists a homomorphism $\mu: L \rightarrow M$ such that $\mu(\rho \pi)=(\mu \rho) \pi \neq 0$. Hence $\mu \rho \neq 0$.

Theorem 3.16. $[\mathbf{5}, \mathbf{8}]$ For a ring $R$ the following conditions are equivalent:

(1) the module $R_{R}$ is FP-injective;

(2) the module ${ }_{R} R$ is a FP-cogenerator;

(3) there is a $f$-flat cogenerator in $R$ Mod;

(4) every left $R$-module is a ( $f$-)submodule of a $f$-flat module;

(5) every $f$ p-injective left $R$-module is a $f$-flat module;

(6) every $f$-injective left $R$-module is a $f$-flat module;

(7) every $f$-flat right $R$-module is a fp-injective module;

(8) every pure-injective $f$-flat right $R$-module is an $f$-injective module.

Remark. The most difficult statements for $F P$-injective rings are proved inside of the category ${ }_{R} \mathrm{C}$ and use localization theory in ${ }_{R} \mathrm{C}$. It would be interesting to have a proof (especially of the equivalence $(1) \Longleftrightarrow(4)$ ) by using usual module-theoretic technique. The author does not know such a proof.

Rings over which any module is embedded into a projective module are quasi-Frobenius. This is the classical Faith-Walker theorem. Rings over which any module is embedded into a flat module, the $I F$-rings, were investigated in the early 70 -s (see, e.g. $[\mathbf{4}, \mathbf{1 4}, \mathbf{2 4}]$ ). The equivalent statements of the preceding theorem are, in a certain sense, similar to properties 
both for QF-rings and for $I F$-rings. We have thus the following proper inclusions:

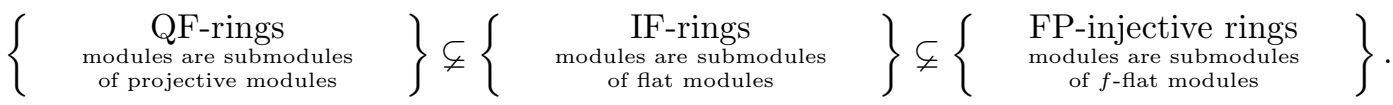

The class of $F P$-injective rings is big enough. For example, given an arbitrary left selfinjective ring $R$ and a locally finite group $G$, the group ring $R(G)$ is left $F P$-injective [5, Theorem 3.2]. Moreover, if $|G|=\infty$, the group $\operatorname{ring} R(G)$ is left $F P$-injective but not left self-injective [5, Corollary 3.3].

\section{The FunCtors $\operatorname{Ext}_{f}$ And $\operatorname{Tor}^{f}$}

Definitions. Let $N$ be a left $R$-module.

(1) The $f$-injective dimension inj. $\operatorname{dim}_{f} N$ is the minimum integer $n$ (if it exists) such that there is a $f$-exact sequence

$$
0 \rightarrow N \rightarrow E^{0} \rightarrow E^{1} \rightarrow \cdots \rightarrow E^{n} \rightarrow 0
$$

with $E^{0}, \cdots, E^{n}$ being $f$-injective modules. We call such a sequence a $f$-resolution of $N$ by $f$-injective modules.

(2) The left global $f$-dimension l.gl. $\operatorname{dim}_{f} R$ of a ring $R$ is $\sup \left\{\operatorname{inj} \cdot \operatorname{dim}_{f} N \mid N \in\right.$ $R \mathrm{Mod}\}$.

(3) The $f p$-injective dimension fp-inj. $\operatorname{dim} M$ is the minimum integer $n$ (if it exists) such that there is a $f$-resolution

$$
0 \rightarrow N \rightarrow E^{0} \rightarrow E^{1} \rightarrow \cdots \rightarrow E^{n} \rightarrow 0
$$

of $N$ by $f p$-injective modules $E^{0}, \cdots, E^{n}$.

If no finite resolution exists, we set inj. $\operatorname{dim}_{f} N$, fp-inj. $\operatorname{dim} N$ equal to $\infty$.

(4) The left global $f p$-dimension l.gl. fp-dim $R$ of a $\operatorname{ring} R$ is $\sup \{f p$-inj. $\operatorname{dim} N \mid N \in$ $R$ Mod\}.

(5) If $M \in R \operatorname{Mod}$, let $\operatorname{Ext}_{f}^{n}(M, N)$ denote $\operatorname{Ext}_{R}^{n} \mathcal{C} / \vec{\delta}^{R}(T M, T N)$.

The abelian groups $\operatorname{Ext}_{f}^{n}(M, N)$ can be computed with the use of $f$-injective $f$-resolutions of $N$. Namely, let

$$
0 \rightarrow N \rightarrow E^{0} \rightarrow E^{1} \rightarrow \cdots
$$

be a $f$-injective $f$-resolution for $N$. Then

$$
0 \rightarrow T N \rightarrow T E^{0} \rightarrow T E^{1} \rightarrow \cdots
$$

is an injective resolution in ${ }_{R} \mathcal{C} / \overrightarrow{\mathcal{S}}^{R}$ for $T N$ by $T E^{i}=-\otimes_{R} E^{i}$ and the cohomology groups for the complex

$$
0 \rightarrow \operatorname{Hom}_{R}\left(M, E^{0}\right) \rightarrow \operatorname{Hom}_{R}\left(M, E^{1}\right) \rightarrow \cdots
$$

are those of the complex

$$
0 \rightarrow\left(T M, T E^{0}\right) \rightarrow\left(T M, T E^{1}\right) \rightarrow \cdots
$$

Any injective resolution for $T N$ in ${ }_{R} \mathrm{C} / \overrightarrow{\mathcal{S}}^{R}$ gives an $f$-injective $f$-resolution for $N$. In a similar way, any $f$-resolution for $N$ by $f p$-injective modules determines a resolution for $T N$ by coh-injective objects in ${ }_{R} \mathcal{C} / \overrightarrow{\mathcal{S}}^{R}$, and vica versa. We also note that any $f$-exact sequence

$$
0 \longrightarrow L \longrightarrow M \longrightarrow N \longrightarrow 0
$$


yields a long exact sequence for any $F \in R \mathrm{Mod}$

$$
\cdots \rightarrow \operatorname{Ext}_{f}^{n-1}(F, N) \rightarrow \operatorname{Ext}_{f}^{n}(F, L) \rightarrow \operatorname{Ext}_{f}^{n}(F, M) \rightarrow \operatorname{Ext}_{f}^{n}(F, N) \rightarrow \cdots
$$

Lemma 4.1. The following are equivalent for a left $R$-module $N$ :

(1) inj. $\operatorname{dim}_{f} N \leqslant n$;

(2) $\operatorname{Ext}_{f}^{p}(M, N)=0$ for all $p>n$ and all $R$-modules $M$;

(3) $\operatorname{Ext}_{f}^{n+1}(M, N)=0$ for all $R$-modules $M$;

(4) if $0 \rightarrow N \rightarrow E^{0} \rightarrow \cdots \rightarrow E^{n-1} \rightarrow L^{n} \rightarrow 0$ is a $f$-resolution of $N$ by $f$-injective modules $E^{i}$, then $L^{n}$ is also $f$-injective.

Proof. Easy.

Corollary 4.2. l.gl. $\operatorname{dim}_{f} R=\sup \left\{n \mid \operatorname{Ext}_{f}^{n}(M, N) \neq 0\right.$ for some $R$-modules $M$ and $\left.N\right\}$.

An acyclic complex $M^{*}=\left(M^{n}, d^{n}\right)$ is said to be $f$-acyclic if each $d^{n}, n \in \mathbf{Z}$, is an $f$-homomorphism. Similarly to the absolute case, the elements of $\operatorname{Ext}_{f}^{n}(M, N), n \geqslant 1$, are represented by $f$-acyclic complexes of the form:

$$
0 \rightarrow N \rightarrow M^{n-1} \rightarrow M^{n-2} \rightarrow \cdots \rightarrow M^{0} \rightarrow M \rightarrow 0
$$

So, the groups $\operatorname{Ext}_{f}^{n}(M, N)$ can be defined alternatively using "Baer addition" on the set of equivalence classes of such complexes (see [11]).

The proof of the following lemma is straightforward (cf. [24, Lemma 3.1]).

Lemma 4.3. The following are equivalent for a left $R$-module $N$ :

(1) fp-inj. $\operatorname{dim} N \leqslant n$;

(2) $\operatorname{Ext}_{f}^{p}(M, N)=0$ for all $p>n$ and all finitely presented $R$-modules $M$;

(3) $\operatorname{Ext}_{f}^{n+1}(M, N)=0$ for all finitely presented $R$-modules $M$;

(4) if $0 \rightarrow N \rightarrow E^{0} \rightarrow \cdots \rightarrow E^{n-1} \rightarrow L^{n} \rightarrow 0$ is a f-resolution of $N$ by fp-injective modules $E^{i}$, then $L^{n}$ is also fp-injective.

It is well-known that a ring $R$ is left Noetherian iff every direct limit of left modules of injective dimension $\leqslant n$ has injective dimension $\leqslant n$. In turn, let FP-inj. $\operatorname{dim} N$ denote the smallest integer $n \geqslant 0$ such that there is a resolution

$$
0 \rightarrow N \rightarrow E^{0} \rightarrow E^{1} \rightarrow \cdots \rightarrow E^{n} \rightarrow 0
$$

of $N$ by $F P$-injective modules $E^{i}$. Then $R$ is left coherent iff every direct limit of left modules of FP-injective dimension $\leqslant n$ has FP-injective dimension $\leqslant n[\mathbf{2 4}$, Theorem 3.2].

Corollary 4.4. Let $R$ be an arbitrary ring. Then every direct limit of left modules of fp-injective dimension $\leqslant n$ has fp-injective dimension $\leqslant n$.

Proof. Let $\left\{M_{i}\right\}_{I}$ be a direct system of left modules of $f p$-injective dimension $\leqslant n$ and let

$$
0 \rightarrow M_{i} \rightarrow E_{i}^{0} \rightarrow E_{i}^{1} \rightarrow \cdots
$$

be the $f$-injective $f$-resolution for $M_{i}, i \in I$, constructed below. Choose the direct system of $f$-injective modules $E_{i}^{0} \supseteq M_{i}$ in the following way. Let $Q$ be an $f$-injective cogenerator. Such a module exists: we can take an injective cogenerator $-\otimes_{R} Q$ in ${ }_{R} \mathcal{C} / \overrightarrow{\mathcal{S}}^{R}$ and then $Q$ is a $f$-injective cogenerator. We set $E_{i}^{0}$ equal to $Q^{\operatorname{Hom}\left(M_{i}, Q\right)}$. The direct system $\left(M_{i}\right)_{I}$ is then extended to a direct system $\left(E_{i}^{0}\right)_{I}$. The modules $E_{i}^{k \geqslant 1}$ are similarly constructed. 
By Lemma 4.3

$$
0 \rightarrow M_{i} \rightarrow E_{i}^{0} \rightarrow \cdots \rightarrow E_{i}^{n-1} \rightarrow L_{i}^{n} \rightarrow 0
$$

is a $f$-resolution of $M_{i}$ by $f p$-injective modules. Since every direct limit of $f p$-injective modules is $f p$-injective, $M$ has a $f$-resolution

$$
0 \rightarrow M \rightarrow \lim _{\longrightarrow} E_{i}^{0} \rightarrow \cdots \rightarrow \lim _{\longrightarrow} E_{i}^{n-1} \rightarrow \lim _{i} L_{i}^{n} \rightarrow 0
$$

by $f p$-injective modules. Hence fp-inj. $\operatorname{dim} M \leqslant n$.

For a left finitely presented module $F$ and a direct system $\left\{M_{i}\right\}_{I}$ of left modules, we consider the canonical homomorphism

$$
\xi_{n}: \lim _{I} \operatorname{Ext}_{f}^{n}\left(F, M_{i}\right) \longrightarrow \operatorname{Ext}_{f}^{n}\left(F, \lim _{I} M_{i}\right) .
$$

Recall that finite presentation of $F$ is equivalent to $\xi_{0}$ being an isomorphism for every $\left\{M_{i}\right\}_{I}[\mathbf{2 5}$, Proposition V.3.4].

Theorem 4.5. $\xi_{n}: \lim _{I} \operatorname{Ext}_{f}^{n}\left(F, M_{i}\right) \longrightarrow \operatorname{Ext}_{f}^{n}\left(F, \lim _{I} M_{i}\right)$ are isomorphisms for all $n \geqslant 0$, for every finitely presented module $F$ and direct system $\left\{M_{i}\right\}_{I}$.

Proof. $\xi_{0}$ is an isomorphim. First we shall show that $\xi_{1}$ is an isomorphism. Let us consider the $f$-injective $f$-resolution

$$
0 \rightarrow M_{i} \rightarrow E_{i}^{0} \stackrel{d_{i}^{0}}{\rightarrow} E_{i}^{1} \stackrel{d_{i}^{1}}{\rightarrow} \ldots
$$

for each $M_{i}$ constructed above.

Let $L_{i}^{0}=E_{i}^{0} / M_{i}$. Since the module $\underline{\lim }_{I} E_{i}^{0}$ is $f p$-injective, it follows that $\operatorname{Ext}_{f}^{1}\left(F, \lim _{I} E_{i}^{0}\right)=$ 0 . We get then the following commutative diagram with exact rows:

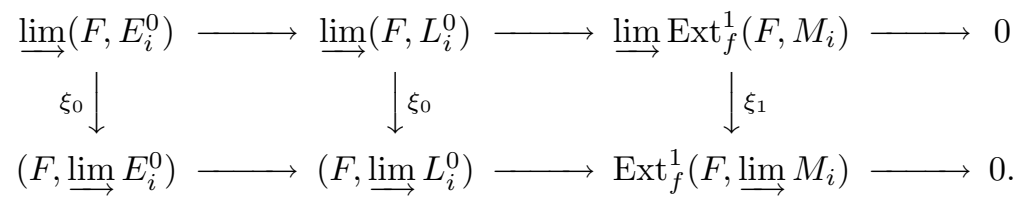

Since the first two arrows of the diagram are isomorphisms, $\xi_{1}$ is an isomorphism as well.

Let $L_{i}^{n}=\operatorname{Im} d_{i}^{n}$. Then $\operatorname{Ext}_{f}^{n}\left(F, M_{i}\right)=\operatorname{Ext}_{f}^{1}\left(F, L_{i}^{n-2}\right), n \geqslant 2$. The sequence

$$
0 \rightarrow \lim _{\longrightarrow} M_{i} \rightarrow \underset{\lim }{\longrightarrow} E_{i}^{0} \rightarrow \lim _{i} E_{i}^{1} \rightarrow \cdots
$$

is a $f$-resolution of $\lim _{i} M_{i}$ by $f p$-injective modules. Then

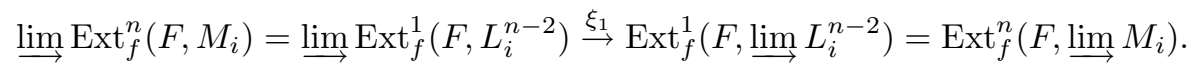

Since $\xi_{1}$ is an isomorphism, it follows that $\xi_{n}$ is an isomorphism.

Stenström [24] showed that $\xi_{n}: \lim _{I} \operatorname{Ext}_{R}^{n}\left(F, M_{i}\right) \longrightarrow \operatorname{Ext}_{R}^{n}\left(F, \lim _{I} M_{i}\right)$ are isomorphisms for all $n \geqslant 0$, for every finitely presented module $F$ and direct system $\left\{M_{i}\right\}_{I}$ iff the ring $R$ is left coherent.

Corollary 4.6. Let $M$ be a left module and $M=\lim _{\longrightarrow} M_{i}$ with $M_{i} \in R \bmod$. Then $\operatorname{Ext}_{f}^{n}(F, M)=\lim _{\longrightarrow} \operatorname{Ext}_{f}^{n}\left(F, M_{i}\right)$ for any finitely presented left module $F$.

A $f$-resolution of a left module $M$ by $f p$-injective modules can be constructed as follows. Let $M=\lim _{\longrightarrow} M_{i}$ be written as a direct limit of finitely presented modules $M_{i}$. Choose for each $i \in I$ the resolution $M_{i} \rightarrow E_{i}^{*}$ of $M_{i}$ by the $f$-injective modules $E_{i}^{*}$ we have constructed above. Then $M \rightarrow \lim _{\longrightarrow} E_{i}^{*}$ is a $f$-resolution of $M$ by $f p$-injective modules. 
Corollary 4.7. The following numbers are the same for any ring $R$ :

(1) l.gl. fp-dim $R$;

(2) $\sup \left\{n \mid \operatorname{Ext}_{f}^{n}(F, M) \neq 0\right.$ for some $F \in R \bmod$ and $\left.M \in R M \operatorname{Mod}\right\}$;

(3) $\sup \{$ fp-inj. $\operatorname{dim} M \mid M \in R \bmod \}$.

Proof. Obviously, l.gl.fp- $\operatorname{dim} R=\sup (2)$ and $\sup (2) \geqslant \sup (3)$. The preceding corollary implies $\sup (3) \geqslant \sup (2)$.

A ring $R$ is said to be almost regular if every (both left and right) module is $f p$-injective; equivalently, $f$-flat (see $[\mathbf{8}]$ ). Since every (both left and right) module is $f p$-injective, every $f$-exact sequence is pure by Proposition 3.11. An almost regular ring is (von Neumann) regular iff it is left or right coherent [8]. Let l. gl. FP-dim $R$ denote $\sup \{$ FP-inj. $\operatorname{dim} M \mid M \in$ $R \operatorname{Mod}\}$.

Obviously, l. gl. FP-dim $R=$ l. gl. fp-dim $R$ when $R$ is left coherent. Also, l.gl.fp-dim $R=$ l. gl. $\operatorname{dim} R$ when $R$ is left Noetherian. By [24, Proposition 3.6] l.gl. FP-dim $R=0$ iff $R$ is (von Neumann) regular. In turn, it follows that l. gl. fp- $\operatorname{dim} R=\mathrm{r}$. gl. fp- $\operatorname{dim} R=0$ iff $R$ is almost regular. The class of non-regular almost regular rings is big enough. Simple almost regular rings we call indiscrete rings $[\mathbf{1 9}, \mathbf{8}]$. Given a non-regular finite dimensional algebra of finite representation type one can construct a non-regular indiscrete ring as a twisted limit of matrix rings $[\mathbf{1 9}, \S 2.4]$. In turn, if $R$ is a non-regular almost regular ring, $G$ a non-trivial locally finite group and the order $|H|$ of every finite subgroup $H$ of $G$ is invertible in $R$, then the group ring $R(G)$ is a non-regular non-indiscrete almost regular ring $[7]$.

There is an interesting problem in our context. A $f$-flat precover of a right module $M$ is a $f$-epimorphism $\varphi: F \rightarrow M$ with $F$ a $f$-flat module such that the induced map $\varphi^{*}: \operatorname{Hom}_{R}\left(F^{\prime}, F\right) \rightarrow \operatorname{Hom}_{R}\left(F^{\prime}, M\right)$ is an epimorphism for any $f$-flat module $F^{\prime}$. We refer to $\varphi$ as a $f$-cover if for every endomorphism $\psi: F \rightarrow F$ the relation $\varphi \psi=\varphi$ implies $\psi$ is an isomorphism.

Question. Every right $R$-module has a $f$-flat (pre-)cover.

Rings over which every module has a $f$-flat cover exist. For example, over an almost regular ring every module is $f$-flat. This is the easiest case. In the absolute case, every module has a flat cover. This has recently been proved by Bican, El Bashir and Enochs in $[\mathbf{2}]$.

Proposition 4.8. For a ring $R$ the following conditions are equivalent:

(1) l. gl. fp- $\operatorname{dim} R \leqslant 1$;

(2) every $f$-quotient module of a $f$-injective module is $f p$-injective;

(3) every $f$-quotient module of a fp-injective module is fp-injective;

(4) if in a short exact sequence $0 \rightarrow M \rightarrow E \rightarrow N \rightarrow 0$ with $M$ a finitely presented module a module $E$ is (FP-)injective, then $N$ is fp-injective.

Proof. Apply Corollary 4.7.

A ring $R$ is left $f$-semihereditary if it satisfies the equivalent conditions of the preceding proposition. 
Corollary 4.9. A left $f$-semihereditary ring $R$ is left semihereditary iff it is left coherent.

Proof. Any left semihereditary ring is left coherent. Conversely, over a left coherent ring the functors $\mathrm{Ext}_{R}^{*}$ and $\mathrm{Ext}_{f}^{*}$ coincide. Now our assertion follows from [23, Proposition 1.22].

If $M=\lim _{\alpha \in I} M_{\alpha}$ with $M_{\alpha}$ finitely presented modules, then there exists a spectral sequence for each module $N$

$$
E_{2}^{p q}=\lim _{I}^{p} \operatorname{Ext}_{R}^{q}\left(M_{\alpha}, N\right) \Longrightarrow \operatorname{Ext}_{R}^{n}(M, N)
$$

In [15, Théorème 4.2], the spectral sequence is constructed from a double complex. Precisely, we construct the pure-exact resolution of $M$

$$
\cdots \stackrel{\partial_{3}}{\longrightarrow} R_{2} \stackrel{\partial_{2}}{\longrightarrow} R_{1} \stackrel{\partial_{1}}{\longrightarrow} R_{0} \stackrel{\partial_{0}}{\longrightarrow} M
$$

where

$$
R_{n}=\bigoplus_{\alpha_{0} \leqslant \alpha_{1} \leqslant \cdots \leqslant \alpha_{n}} M_{\alpha_{0}, \alpha_{1}, \cdots, \alpha_{n}}
$$

and $M_{\alpha_{0}, \alpha_{1}, \cdots, \alpha_{n}}$ is a copy of $M_{\alpha_{0}}$.

Let

$$
N \longrightarrow E^{0} \stackrel{\partial^{0}}{\longrightarrow} E^{1} \stackrel{\partial^{1}}{\longrightarrow} E^{2} \stackrel{\partial^{2}}{\longrightarrow} \cdots
$$

be an injective resolution of $N$. Then the bicomplex is

$$
E_{0}^{p q}=\operatorname{Hom}_{R}\left(R_{p}, E^{q}\right),
$$

where the vertical and horizontal differentials are

$$
d_{0}^{p q}=(-1)^{p} \operatorname{Hom}_{R}\left(R_{p}, \partial^{q}\right): E_{0}^{p q} \longrightarrow E_{0}^{p, q+1}
$$

and

respectively.

$$
d_{1}^{p q}=(-1)^{p} \operatorname{Hom}_{R}\left(\partial_{p+1}, E^{q}\right): E_{0}^{p q} \longrightarrow E_{0}^{p+1, q},
$$

If we replace the injective resolution $N \rightarrow E^{*}$ by an $f$-injective $f$-resolution of $N$ and also apply Corollary 4.6, we shall get the following result.

Theorem 4.10. Let $M$ and $N$ be two modules, $M=\lim _{\alpha} M_{\alpha}$ and $N=\lim _{\beta} N_{\beta}$ with $M_{\alpha}$ and $N_{\beta}$ finitely presented modules. Then the following relation is valid:

$$
\varliminf_{I}^{p} \lim _{J} \operatorname{Ext}_{f}^{q}\left(M_{\alpha}, N_{\beta}\right)=\varliminf_{I}^{p} \operatorname{Ext}_{f}^{q}\left(M_{\alpha}, N\right) \Longrightarrow \operatorname{Ext}_{f}^{n}(M, N) \text {. }
$$

Suppose $M$ has a $f$-resolution by $f$-flat modules $F_{*} \rightarrow M$. Given a left module $N$ we put

$$
\operatorname{Tor}_{*}^{f}(M, N)=H_{*}\left(F_{*} \otimes_{R} N\right) .
$$

Any $f$-exact sequence of left $R$-modules

$$
0 \longrightarrow L \longrightarrow M \longrightarrow N \longrightarrow 0
$$

yields a long exact sequence for any $G \in \operatorname{Mod} R$ having a $f$-flat $f$-resolution

$$
\cdots \rightarrow \operatorname{Tor}_{n+1}^{f}(G, N) \rightarrow \operatorname{Tor}_{n}^{f}(G, L) \rightarrow \operatorname{Tor}_{n}^{f}(G, M) \rightarrow \operatorname{Tor}_{n}^{f}(G, N) \rightarrow \cdots
$$

Remark. The definition of the functor $\operatorname{Tor}^{f}$ depends on the choice of a $f$-flat $f$-resolution. In the absolute case, the groups $\operatorname{Tor}^{R}$ do not depend on the choice of a flat resolution (see e.g. [28]) since every module admits a projective resolution. It is not clear, however, whether the class $\omega_{f}$ is projective. The case of almost regular rings is trivial, because $\operatorname{Tor}_{n}^{f} \equiv 0$ for all $n \geqslant 1$. 
We consider the duality homomorphisms

$$
\rho: \operatorname{Ext}_{R}^{n}(M, \widehat{N}) \longrightarrow \operatorname{Tor}_{n}^{R}(M, N)^{\wedge}
$$

and

$$
\sigma: \operatorname{Tor}_{n}^{R}(\widehat{N}, F) \longrightarrow \operatorname{Ext}_{R}^{n}(F, N)^{\curlywedge}
$$

where $M \in \operatorname{Mod} R, F, N \in R \operatorname{Mod}$. The first homomorphism is always an isomorphism [3, Proposition VI.5.1] whereas $\sigma$ is an isomorphism for every finitely generated module $F$ whenever $R$ is a left Noetherian [3, Proposition VI.5.3] or for every finitely presented module $F$ whenever $R$ is a left coherent [23, Lemma 1.12].

It is not clear, however, whether a homomorphism $\widehat{\mu}$ is a $f$-homomorphism if $\mu$ is a $f$ homomorphism. Therefore we specify some classes of modules to construct the analogous duality homomorphisms corresponding to the functors $\operatorname{Ext}_{f}$ and $\operatorname{Tor}^{f}$. Precisely, let $\mathcal{F}(R)$ $\left(\mathcal{F}\left(R^{\mathrm{op}}\right)\right)$ denote the subcategory of right (left) modules $M$ that have a $f$-flat $f$-resolution $F_{*} \rightarrow M$ such that $\widehat{M} \rightarrow \widehat{F}^{*}$ is a $f$-injective $f$-resolution for the character module $\widehat{M}$. The class $\mathcal{F}(R)$ is non-empty, because every $f p$-flat module belongs to $\mathcal{F}(R)$. In a similar way, $\mathcal{J}\left(R^{\mathrm{op}}\right)(\mathrm{J}(R))$ is the subcategory of left (right) modules $M$ that have $f$-injective $f$-resolution $M \rightarrow E^{*}$ such that $\widehat{E}_{*} \rightarrow \widehat{M}$ is a $f$-flat $f$-resolution for the character module $\widehat{M}$. Obviously the $f p$-injective left modules belong to $\mathcal{J}\left(R^{\mathrm{op}}\right)$.

Proposition 4.11. For a right FP-injective ring $R$ and $M \in \mathcal{J}\left(R^{\mathrm{op}}\right)$ the following are equivalent:

(1) fp-inj. $\operatorname{dim} M=0$;

(2) fp-inj. $\operatorname{dim} M \leqslant n$.

Proof. The implication (1) $\Longrightarrow(2)$ is trivial. Let us show (2) $\Longrightarrow(1)$. Let $M \rightarrow E^{*}$ be a $f$-injective $f$-resolution for $M$ such that $\widehat{E}_{*} \rightarrow \widehat{M}$ is a $f$-flat $f$-resolution for $\widehat{M}$. Then the module $K^{n}=\operatorname{Im} d^{n-1}$ in the exact sequence

$$
0 \rightarrow M \rightarrow E^{0} \rightarrow \cdots \rightarrow E^{n-1} \stackrel{d^{n-1}}{\rightarrow} K^{n} \rightarrow 0
$$

is $f p$-injective. It is enough to show that $L^{n-1}=\operatorname{Ker} d^{n-1}$ is $f p$-injective. By assumption, the sequence

$$
0 \rightarrow \widehat{K}^{n} \rightarrow \widehat{E}^{n-1} \rightarrow \cdots \rightarrow \widehat{E}^{0} \rightarrow \widehat{M} \rightarrow 0
$$

is a $f$-flat $f$-resolution of $\widehat{M}$. By Theorem 3.16 all the $E^{i}, i<n$, and the $K^{n}$ are $f$-flat modules. Therefore $\widehat{K}^{n}$ is a direct summand of $\widehat{E}^{n-1}$ by Corollary 3.9. Hence $\widehat{L}^{n-1}$ is a direct summand of $\widehat{E}^{n-1}$ as well. This implies $\widehat{L}^{n-1}$ is $f$-flat, and $L^{n-1}$ is $f p$-injective.

Given modules $M \in \operatorname{Mod} R$ and $N \in \mathcal{F}\left(R^{\text {op }}\right)$ we consider the duality homomorphism

$$
\rho: \operatorname{Ext}_{f}^{n}(M, \widehat{N}) \longrightarrow \operatorname{Tor}_{n}^{f}(M, N) \text {. }
$$

Similar to the absolute case [3, Proposition VI.5.1] $\rho$ is an isomorphism. It follows that given two $f$-flat $f$-resolutions for $N$ we obtain the same functors $\operatorname{Tor}_{n}^{f}(-, N)$. This follows from the fact that the functors $\operatorname{Ext}_{f}^{n}(-, \widehat{N})$ do not depend on the choice of a $f$-injective $f$-resolution for $\widehat{N}$ and that $\rho$ is an isomorphism.

Let $F \in R \operatorname{Mod}$ and $N \in \mathcal{J}\left(R^{\mathrm{op}}\right)$. There is a $f$-injective $f$-resolution $N \rightarrow E^{*}$ for $N$ such that $\widehat{E}_{*} \rightarrow \widehat{N}$ is a $f$-flat $f$-resolution for the character module $\widehat{N}$. In this case we consider 
the groups $\operatorname{Tor}_{n}^{f}(\widehat{N}, F)$ relative to this resolution. There is a duality homomorphism

$$
\sigma: \operatorname{Tor}_{n}^{f}(\widehat{N}, F) \longrightarrow \operatorname{Ext}_{f}^{n}(F, N) \widehat{.}
$$

If $F$ is finitely presented, then each natural homomorphism $\widehat{E}^{i} \otimes_{R} F \rightarrow \operatorname{Hom}_{R}\left(F, E^{i}\right)^{\curlyvee}$ with $E^{i}$ the $i$ th component of the complex $E^{*}, i \geqslant 0$, is an isomorphism. We see that $\sigma$ is an isomorphism whenever $F$ is finitely presented.

Now let $F$ be a finitely generated left module without finite presentations, $0 \rightarrow H \rightarrow P \stackrel{\varphi}{\rightarrow}$ $F \rightarrow 0$ an exact sequence with $P$ a finitely generated free module. Then $F=\lim _{\longrightarrow} F_{\alpha}$ where $F_{\alpha}=P / H_{\alpha}, H_{\alpha}$ are finitely generated submodules of $H$. Moreover, $\varphi=\underline{\lim } \overrightarrow{\varphi_{\alpha}}$ where $\varphi_{\alpha}$ are epimorphisms $P \rightarrow F_{\alpha}$. Let $G$ denote the kernel $\operatorname{Ker} T \varphi, G_{\alpha}$ the kernel $\operatorname{Ker} T \varphi_{\alpha}$ and $D_{\alpha}=G / G_{\alpha}$.

Lemma 4.12. If a left module $F$ is finitely generated without finite presentations and $N$ is a $f$-injective module, then the kernel of the natural homomorphism $\widehat{N} \otimes_{R} F \rightarrow \operatorname{Hom}_{R}(F, N)^{\wedge}$ is the abelian group $\lim _{\longrightarrow} \operatorname{Hom}_{R} \mathrm{e} / \overrightarrow{\mathcal{s}}_{R}\left(D_{\alpha}, T N\right)^{\curlywedge}=\lim _{\longrightarrow} \operatorname{Hom}_{R} \mathrm{e} / \overrightarrow{\mathcal{s}}^{R}\left(D_{\alpha},-\otimes_{R} N\right) \uparrow$.

Proof. Indeed, for each $\alpha$ we have an exact sequence $0 \rightarrow D_{\alpha} \rightarrow T F_{\alpha} \rightarrow T F \rightarrow 0$. If $N$ is a $f$-injective module, then the sequence

$$
0 \longrightarrow\left(D_{\alpha}, T N\right)^{\wedge} \longrightarrow\left(T F_{\alpha}, T N\right)^{\curlywedge} \longrightarrow(T F, T N)^{\curlywedge} \longrightarrow 0
$$

is exact. Since the functor $T$ is fully faithful and the functor of direct limit is exact, we get the exact sequence

$$
0 \longrightarrow \lim _{\longrightarrow}\left(D_{\alpha}, T N\right)^{\curlywedge} \longrightarrow \lim _{\longrightarrow} \operatorname{Hom}_{R}\left(F_{\alpha}, N\right)^{\curlywedge} \longrightarrow \operatorname{Hom}_{R}(F, N)^{\curlywedge} \longrightarrow 0 .
$$

All the modules $F_{\alpha}$ are finitely presented, and so all the groups $\operatorname{Hom}_{R}\left(F_{\alpha}, N\right)^{\wedge}$ are isomorphic to $\widehat{N} \otimes_{R} F_{\alpha}$. It remains to observe that $\underline{\lim } \widehat{N} \otimes_{R} F_{\alpha}=\widehat{N} \otimes_{R} F$.

Theorem 4.13 (The Sklyarenko exact sequence). If $F$ is a finitely generated left module, then for any module $N \in \mathcal{J}\left(R^{\mathrm{op}}\right)$ the duality homomorphism $\sigma$ fits into the exact sequence

$$
\begin{aligned}
& \cdots \stackrel{\sigma}{\rightarrow} \operatorname{Ext}_{f}^{n+1}(F, N) \stackrel{\delta}{\rightarrow} \varliminf_{\lim } \operatorname{Ext}_{R}^{n} \mathrm{e} / \overrightarrow{\mathcal{S}}^{R}\left(D_{\alpha}, T N\right)^{\curlywedge} \rightarrow \operatorname{Tor}_{n}^{f}(\widehat{N}, F) \rightarrow \\
& \stackrel{\sigma}{\rightarrow} \operatorname{Ext}_{f}^{n}(F, N)^{\curlywedge} \rightarrow \cdots \stackrel{\delta}{\rightarrow} \lim _{\longrightarrow} \operatorname{Hom}_{R} \mathcal{e} / \overrightarrow{\mathcal{S}}_{R}\left(D_{\alpha}, T N\right)^{\curlywedge} \rightarrow \widehat{N} \otimes_{R} F \stackrel{\sigma}{\rightarrow} \operatorname{Hom}_{R}(F, N)^{\curlywedge} \rightarrow 0 .
\end{aligned}
$$

Proof. Let $N \rightarrow Y^{*}$ be a $f$-injective $f$-resolution of $N$. By the preceding lemma the sequence of complexes

$$
0 \longrightarrow \lim _{(}\left(D_{\alpha}, T Y^{*}\right)^{\curlywedge} \longrightarrow \widehat{Y}_{*} \otimes_{R} F \longrightarrow \operatorname{Hom}_{R}\left(F, Y^{*}\right)^{\curlywedge} \longrightarrow 0
$$

is exact. The sequence of the theorem is the homological sequence that corresponds to (4.1).

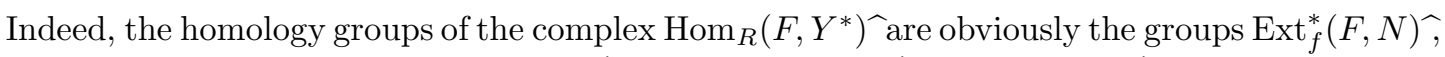
the homology groups of the complex $\widehat{Y}_{*} \otimes_{R} F$ are $\operatorname{Tor}_{*}^{f}(\widehat{N}, F)$, because $\widehat{Y}_{*}$ is a $f$-flat $f$ resolution of $\widehat{N}$, by assumption. Finally,

$$
\begin{aligned}
& H_{n}\left(\lim _{\longrightarrow}\left(D_{\alpha}, T Y^{*}\right)^{\wedge}\right)=\lim _{\longrightarrow} H_{n}\left(\left(D_{\alpha}, T Y^{*}\right)^{\wedge}\right)= \\
& =\underline{\lim _{\longrightarrow}} H^{n}\left(\left(D_{\alpha}, T Y^{*}\right)\right)^{\curlywedge}=\underline{\lim _{\longrightarrow}} \operatorname{Ext}_{R}^{n} \mathrm{e} / \overrightarrow{\mathcal{S}}^{R}\left(D_{\alpha}, T N\right)^{\wedge}
\end{aligned}
$$

because the homology functor $H_{n}$ commutes with the exact functors $\stackrel{\lim }{\longrightarrow}$ and $X \rightarrow \widehat{X}$. 
Now we suppose that $R$ is a left coherent ring, and let $F$ be a finitely generated left module and $0 \rightarrow G \rightarrow P \rightarrow F \rightarrow 0$ an exact sequence with $P$ a finitely generated free module. Then $F=\underline{\lim } F_{\alpha}$ where $F_{\alpha}=P / G_{\alpha}$ and $G_{\alpha}$ are finitely presented submodules of $P$. As above, let $D_{\alpha}$ denote $G / G_{\alpha}$.

Corollary 4.14. [23] If $R$ is a left coherent ring, $F$ is a finitely generated left module, then for any module $N \in R$ Mod the duality homomorphism $\sigma$ fits into an exact sequence

$$
\begin{aligned}
& \cdots \stackrel{\sigma}{\rightarrow} \operatorname{Ext}_{R}^{n+1}(F, N)^{\wedge} \stackrel{\delta}{\rightarrow} \underset{\lim _{\longrightarrow}}{\longrightarrow} \operatorname{Ext}_{R}^{n}\left(D_{\alpha}, N\right)^{\curlywedge} \rightarrow \operatorname{Tor}_{n}^{R}(\widehat{N}, F) \rightarrow \\
& \quad \stackrel{\sigma}{\rightarrow} \operatorname{Ext}_{R}^{n}(F, N)^{\curlywedge} \rightarrow \cdots \stackrel{\delta}{\rightarrow} \lim _{\longrightarrow} \operatorname{Hom}_{R}\left(D_{\alpha}, N\right)^{\curlywedge} \rightarrow \widehat{N} \otimes_{R} F \stackrel{\sigma}{\rightarrow} \operatorname{Hom}_{R}(F, N)^{\curlywedge} \rightarrow 0 .
\end{aligned}
$$

Proof. Since over a left coherent ring the character module of any injective module is flat, the proof is similar to that of Theorem 4.13.

\section{The Derived Categories $D_{f}(R)$ And $D\left({ }_{R} \mathcal{C} / \overrightarrow{\mathcal{S}}^{R}\right)$}

In this section we use Generalov's construction of the relative derived category (see [11]) to describe the derived category of the locally coherent Grothendieck category ${ }_{R} \mathcal{C} / \overrightarrow{\mathcal{S}}^{R}$.

Let $K(R)$ and $K\left({ }_{R} \mathcal{C} / \overrightarrow{\mathcal{S}}^{R}\right)$ denote the corresponding homotopy categories of $R$ Mod and ${ }_{R} \mathcal{C} / \overrightarrow{\mathcal{S}}^{R}$, i.e. the quotient categories of $\operatorname{Kom}(R)$ and $\operatorname{Kom}\left({ }_{R} \mathcal{C} / \overrightarrow{\mathcal{S}}^{R}\right)$ respectively modulo homotopy equivalence. The functor $T$ induces the fully faithful embeddings

$$
\operatorname{Kom}(R) \longrightarrow \operatorname{Kom}\left({ }_{R} \mathrm{C} / \overrightarrow{\mathcal{S}}^{R}\right)
$$

and

$$
K(R) \longrightarrow K\left({ }_{R} \mathrm{C} / \overrightarrow{\mathcal{S}}^{R}\right)
$$

that take a complex $M^{*}=\left(M^{n}, d^{n}\right)$ to $T M^{*}=\left(T M^{n}, T d^{n}\right)$. We recall that a mapping cone of a morphism $\mu: M^{*} \rightarrow N^{*}$ in $\operatorname{Kom}(R)$ is defined as the complex

$$
C(\mu)=\left(M^{n+1} \bigoplus N^{n}, d_{C(\mu)}\right), \quad d_{C(\mu)=}\left(\begin{array}{cc}
-d_{M} & 0 \\
\mu & d_{N}
\end{array}\right)
$$

We have the following sequence of complexes

$$
M \stackrel{\mu}{\longrightarrow} N \stackrel{\nu}{\longrightarrow} C(\mu) \stackrel{\rho}{\longrightarrow} M[1]
$$

where $(M[1])^{n}=M^{n+1}, \nu^{n}: N^{n} \rightarrow C(\mu)^{n}$ and $\rho^{n}: C(\mu)^{n} \rightarrow M^{n+1}$ are the canonical injection and projection respectively. The family of such sequences (up to isomorphism the so-called "distinguish triangles") defines the structure of a triangulated category on the homotopy category $K(R)$ (see $[\mathbf{2 7}, \mathbf{1 0}, \mathbf{2 8}]$ ).

A morphism $\mu: X^{*} \rightarrow Y^{*}$ in $K(R)$ is called a $f$-quasi-isomorphism if its mapping cone $C(\mu)$ is $f$-acyclic. Obviously, if $\mu$ is a $f$-quasi-isomorphism then $T \mu$ is a quasi-isomorphism in $K\left({ }_{R} \mathcal{C} / \overrightarrow{\mathcal{S}}^{R}\right)$. We denote by $\mathcal{S}_{f}$ the class of all $f$-quasi-isomorphisms in $K(R)$.

Theorem 5.1 (Generalov [11]). The class of $f$-quasi-isomorphisms is localising in $K(R)$.

We can thus construct the localization of $K(R)$ with respect to $\mathcal{S}_{f}$, and we define the relative derived category $D_{f}(R)$ of $R \operatorname{Mod}$ as this localization: $D_{f}(R)=K(R)\left[\mathcal{S}_{f}\right]$ (see [11]). The category $D_{f}(R)$ inherits the structure of a triangulated category from $K(R)$. It is easy to see that the functor $T$ induces an embedding of $D_{f}(R)$ into the derived category $D\left({ }_{R} \mathcal{C} / \overrightarrow{\mathcal{S}}^{R}\right)$ of ${ }_{R} \mathcal{C} / \overrightarrow{\mathcal{S}}^{R}$. If we start with the homotopy category $K^{+}(R)$ (respectively $K^{-}(R)$ or $K^{b}(R)$ ) of 
complexes bounded from below (respectively bounded from above or bounded complexes), then we get in a similar way the derived categories $D_{f}^{+}(R)$ (respectively $D_{f}^{-}(R)$ or $D_{f}^{b}(R)$ ).

Let $K^{+}\left(\mathcal{J}_{f}\right)$ denote the homotopy category of complexes bounded from below over the full subcategory $\mathcal{J}_{f}$ of $R$ Mod consisting of $f$-injectives. Generalov has shown [11] that the natural functor $\Phi: K^{+}\left(\mathcal{J}_{f}\right) \rightarrow D_{f}^{+}(R)$ that takes $E^{*} \in K^{+}\left(\mathcal{J}_{f}\right) \subseteq K^{+}(R)$ to its image in $D_{f}^{+}(R)$ is an equivalence of categories. Obviously, the functor

$$
T: K^{+}\left(\mathcal{J}_{f}\right) \longrightarrow K^{+}(\mathcal{J}), \quad E^{*} \mapsto T E^{*}
$$

is an equivalence of the category $K^{+}\left(\mathcal{J}_{f}\right)$ and the homotopy category of complexes bounded from below over the full subcategory $\mathcal{J}$ of ${ }_{R} \mathcal{C} / \overrightarrow{\mathcal{S}}^{R}$ consisting of ${ }_{R} \mathrm{C} / \overrightarrow{\mathcal{S}}^{R}$-injectives. By $[\mathbf{1 0}$, Theorem 3.5.21] the natural functor $\widetilde{\Phi}: K^{+}(\mathcal{J}) \rightarrow D^{+}\left({ }_{R} \mathcal{C} / \overrightarrow{\mathcal{S}}^{R}\right)$ is an equivalence of categories. Let $\Psi$ denote the equivalence of categories quasi-inverse to $\Phi$.

Theorem 5.2. The derived categories $D_{f}^{+}(R)$ and $D^{+}\left({ }_{R} \mathrm{C} / \overrightarrow{\mathcal{S}}^{R}\right)$ are naturally equivalent via the functor $\widetilde{\Phi} \circ T \circ \Psi$.

Let the functor $I_{f}$ send a module $M \in R$ Mod to the complex $\cdots 0 \rightarrow M \rightarrow 0 \cdots$ concentrated at zero degree. Then the following relation holds:

$$
\operatorname{Ext}_{f}^{n}(M, N)=\operatorname{Hom}_{D_{f}(R)}\left(I_{f}(M), I_{f}(N)[n]\right)
$$

where $M, N \in R \operatorname{Mod}[\mathbf{1 1}]$. The groups $\operatorname{Ext}_{f}^{n}(M, N)$ can also be defined [11] by using "Baer addition" on the set of equivalence classes of $f$-acyclic complexes of the form:

$$
0 \rightarrow N \rightarrow M^{n-1} \rightarrow \cdots \rightarrow M^{0} \rightarrow M \rightarrow 0 .
$$

On the other hand, let the functor $I$ take every $M \in R \operatorname{Mod}$ to the complex $\cdots 0 \rightarrow T M \rightarrow$ $0 \cdots$ Then

$$
\begin{aligned}
& \left.\operatorname{Ext}_{f}^{n}(M, N)=\operatorname{Hom}_{D\left({ }_{R}\right.} \mathcal{C} / \overrightarrow{\mathcal{S}}^{R}\right) \\
& \text { 6. } K \text {-Groups FOR THE CATEGORY } \operatorname{coh}{ }_{R} \mathcal{C} / \overrightarrow{\mathcal{S}}^{R}
\end{aligned}
$$

An exact category $\mathcal{C}$ is a full subcategory of an abelian category $\mathcal{A}$ which is closed under extensions and which contains a zero object of $\mathcal{A}$. A sequence $0 \rightarrow E^{\prime} \rightarrow E \rightarrow E^{\prime \prime} \rightarrow 0$ in $\mathcal{C}$ is called exact if it is exact in $\mathcal{A}$. We say that a map $i: M \rightarrow N$ in an exact category $\mathcal{C}$ is an admissible monomorphism if there is a short exact sequence $0 \rightarrow M \rightarrow N \rightarrow L \rightarrow 0$ in $\mathcal{C}$. In other words, the cokernel of $i$ in the abelian category $\mathcal{A}$ is in $\mathcal{C}$. Similarly $j: M \rightarrow N$ in an exact category $\mathcal{C}$ is an admissible epimorphism if there is a short exact sequence $0 \rightarrow L \rightarrow M \rightarrow N \rightarrow 0$ in $\mathcal{C}$.

If $\mathcal{M}$ is an exact category define $Q \mathcal{N}$ to be the category with the same objects as $\mathcal{N}$ and with morphisms defined as follows. A morphism from $M$ to $N$ in $Q \mathcal{M}$ is an equivalence class of diagrams of the form

$$
M \nleftarrow X \longmapsto N
$$

in $\mathcal{M}$. Here $\rightarrow$ denotes an admissible epimorphism (with kernel in $\mathcal{M}$ ) and $\longmapsto$ denotes an admissible monomorphism (with cokernel in $\mathcal{M}$ ). We say that $M \nleftarrow X \mapsto N$ and $M \nleftarrow Y \longmapsto N$ are equivalent if there is an isomorphism $X \simeq Y$ making

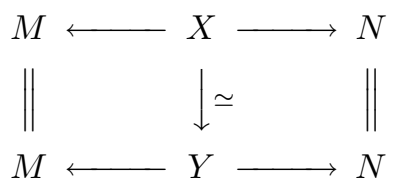


commutative (see $[\mathbf{2 0}, \mathbf{2 6}]$ ).

Theorem 6.1 (Resolution). Let $\mathcal{C}$ be an exact category an let $\mathcal{M} \subseteq \mathcal{C}$ be a full subcategory of $\mathcal{C}$ closed under extensions. Assume

(1) if $0 \rightarrow M^{\prime} \rightarrow M \rightarrow M^{\prime \prime} \rightarrow 0$ is a short exact sequence in $\mathcal{C}$ with $M^{\prime}$ and $M$ in $\mathcal{M}$, then $M^{\prime \prime}$ is in $\mathcal{M}$;

(2) for every object $C$ in $\mathcal{C}$ there is a finite resolution

$$
0 \longrightarrow C \longrightarrow M_{0} \longrightarrow M_{1} \longrightarrow \cdots \longrightarrow M_{n} \longrightarrow 0
$$

with $M_{i}$ in $\mathcal{M}$.

Then $K_{n}(\mathcal{M}) \stackrel{\simeq}{\longrightarrow} K_{n}(\mathrm{e})$.

Lemma 6.2. For any $C \in \operatorname{coh}_{R} \mathcal{C} / \overrightarrow{\mathcal{S}}^{R}$ there is an exact sequence

$$
0 \longrightarrow C \stackrel{i}{\longrightarrow} T M \stackrel{T \mu}{\longrightarrow} T N \longrightarrow T L \longrightarrow 0
$$

with $M, N$ and $L$ finitely presented modules.

Proof. By [13, Theorem 2.16] there exists a coherent object $D$ of $\operatorname{coh}_{R} \mathcal{C}$ such that $C=D_{\mathcal{S}^{R}}$. The object $D$ fits into an exact sequence

$$
0 \longrightarrow D \longrightarrow-\otimes_{R} M \stackrel{-\otimes \mu}{\longrightarrow}-\otimes_{R} N \longrightarrow-\otimes_{R} L \longrightarrow 0
$$

with $M, N, L \in R \bmod$. If we apply the exact functor of $\mathcal{S}^{R}$-localization to this sequence, we shall obtain the exact sequence of lemma.

Lemma 6.3. Let $\mathcal{M}=\{T M \mid M \in R \bmod \}$. Then $\mathcal{M}$ is closed under extensions in $\operatorname{coh}_{R} \mathrm{C} / \overrightarrow{\mathcal{S}}^{R}$.

Proof. Let

$$
0 \longrightarrow T M \longrightarrow C \longrightarrow T N \longrightarrow 0
$$

be an exact sequence in $\operatorname{coh}_{R} \mathrm{C} / \overrightarrow{\mathcal{S}}^{R}$ with $M$ and $N$ finitely presented. Then the module $K=C(R)$ is finitely presented. By the preceding lemma there is an exact sequence

$$
0 \longrightarrow C \stackrel{i}{\longrightarrow} T L \stackrel{T \mu}{\longrightarrow} T E
$$

with $L$ and $E$ finitely presented modules. Then $K=\operatorname{Ker} \mu$ and there is a unique morphism $\varphi: T K \rightarrow C$ such that $T \rho=i \varphi$ with $\rho=\operatorname{ker} \mu$. We claim that $\varphi$ is an isomorphism.

The module $F=L / K$ is finitely presented and the exact sequense

$$
0 \longrightarrow K \longrightarrow L \longrightarrow F \longrightarrow 0
$$

is $f$-exact. We have a commutative diagram with exact rows:

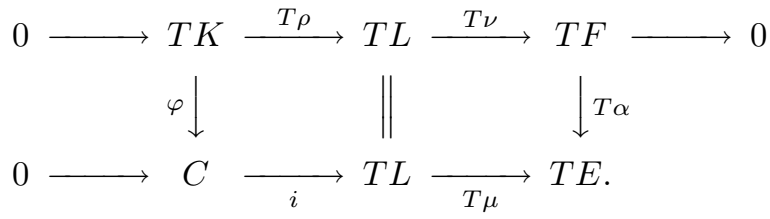

Since $F$ is a finitely presented submodule of $E$, it follows that $T \alpha$ is a monomorphism. By the snake lemma $\varphi$ is an isomorphism and, hence, $C \in \mathcal{M}$.

$G$-theory of a ring $R$ is, by definition, the $K$-theory of the exact category of finitely presented modules $R$ mod. Preceding two lemmas imply the following. 
Proposition 6.4. The subcategory $\mathcal{M}=T(R \bmod )$ of $\mathcal{C}$ satisfies all the hypotheses of the Resolution Theorem. In particular, the functor $T$ induces an isomorphism of $K$-groups $G_{n}(R) \stackrel{\simeq}{\longrightarrow} K_{n}\left(\operatorname{coh}_{R} \mathrm{e} / \overrightarrow{\mathcal{S}}^{R}\right), n \geqslant 0$.

Let ${ }^{R} \mathcal{S}=\left\{C \in \operatorname{coh} \mathcal{C}_{R} \mid C(R)=0\right\}$. The Auslander-Gruson-Jensen duality $D$ takes the category ${ }^{R} \mathcal{S}$ to $\mathcal{S}_{R}$. By $[\mathbf{1 3}$, Theorem 5.5] $D$ induces a duality

$$
D: \operatorname{coh} \mathcal{C}_{R} /{ }^{R} \overrightarrow{\mathcal{S}} \longrightarrow \operatorname{coh}_{R} \mathcal{C} / \overrightarrow{\mathcal{S}}_{R}
$$

Then the classifying spaces for the categories $Q \operatorname{coh} \mathcal{C}_{R} /{ }^{R} \overrightarrow{\mathcal{S}}$ and $Q \operatorname{coh}{ }_{R} \mathrm{C} / \overrightarrow{\mathcal{S}}_{R}$ are homeomorphic.

Proposition 6.5. The functors $T$ and $D$ induce an isomorphism of $K$-groups $G_{n}\left(R^{\mathrm{op}}\right) \stackrel{\simeq}{\longrightarrow}$ $K_{n}\left(\operatorname{coh}_{R} \mathcal{C} / \overrightarrow{\mathcal{S}}_{R}\right), n \geqslant 0$.

Corollary 6.6. If $R$ is a left and right $F P$-injective ring, then the functors $T$ and $D$ induce an isomorphism of $K$-groups $G_{n}\left(R^{\mathrm{op}}\right) \stackrel{\simeq}{\longrightarrow} G_{n}(R), n \geqslant 0$.

Proof. A ring $R$ is left and right $F P$-injective iff $\mathcal{S}^{R}=\mathcal{S}_{R}$ [8, Theorem 2.5]. Therefore our assertion follows from the preceding two propositions.

The duality $D$ yields isomorophisms of $K$-groups $K_{i}\left(\operatorname{coh}_{R} \mathrm{C}\right) \stackrel{\simeq}{\longrightarrow} K_{i}\left(\operatorname{coh} \mathcal{C}_{R}\right)$. Since every coherent object $C \in \operatorname{coh}_{R} \mathcal{C}$ fits into an exact sequence

$$
0 \rightarrow C \rightarrow-\otimes_{R} M \rightarrow-\otimes_{R} N \rightarrow-\otimes_{R} L \rightarrow 0
$$

the Resolution theorem implies that the $K$-theory of $\operatorname{coh}_{R} \mathrm{C}$ is equivalent to the $K$-theory of $R \bmod ^{\oplus}$. Here $R \bmod ^{\oplus}$ denotes the exact category of finitely presented left $R$-modules where only short exact sequences are used. Let ${ }_{R} \mathcal{S}$ denote the Serre subcategory $D \mathcal{S}^{R}=$ $\left\{C \in \operatorname{coh} \mathcal{C}_{R} \mid\left(C, R \otimes_{R}-\right)=0\right\}$ of $\operatorname{coh} \mathcal{C}_{R}$.

Proposition 6.7. The functors $T$ and $D$ induce two isomorphic long exact sequences

$$
\begin{aligned}
& \cdots G_{n+1}(R) \stackrel{\delta}{\rightarrow} K_{n}\left(\mathcal{S}^{R}\right) \rightarrow K_{n}\left(R \bmod ^{\oplus}\right) \rightarrow G_{n}(R) \rightarrow \\
& \cdots \stackrel{\delta}{\rightarrow} K_{0}\left(\mathcal{S}^{R}\right) \rightarrow K_{0}\left(R \bmod ^{\oplus}\right) \rightarrow G_{0}(R) \rightarrow 0
\end{aligned}
$$

and

$$
\begin{aligned}
\cdots G_{n+1}(R) \stackrel{\delta}{\rightarrow} K_{n}\left({ }_{R} \mathcal{S}\right) \rightarrow K_{n}\left(\bmod ^{\oplus} R\right) & \rightarrow G_{n}(R) \rightarrow \\
& \ldots \stackrel{\delta}{\rightarrow} K_{0}\left({ }_{R} \mathcal{S}\right) \rightarrow K_{0}\left(\bmod ^{\oplus} R\right) \rightarrow G_{0}(R) \rightarrow 0 .
\end{aligned}
$$

Proof. Since $\mathcal{S}^{R}$ is a Serre subcategory of $\operatorname{coh}_{R} \mathcal{C}$, by $[\mathbf{2 0}$, Theorem 5.5] we have a long exact sequence

$$
\cdots \longrightarrow K_{1}\left(\operatorname{coh}_{R} \mathcal{C} / \overrightarrow{\mathcal{S}}^{R}\right) \stackrel{\delta}{\longrightarrow} K_{0}\left(\mathcal{S}^{R}\right) \longrightarrow K_{0}\left(R \bmod ^{\oplus}\right) \longrightarrow K_{0}\left(\operatorname{coh}_{R} \mathcal{C} / \overrightarrow{\mathcal{S}}^{R}\right) \longrightarrow 0 .
$$

By Proposition $6.4 G_{n}(R)$ is isomorphic to $K_{n}\left(\operatorname{coh}_{R} \mathcal{C} / \overrightarrow{\mathcal{S}}^{R}\right)$ for all $n \geqslant 0$. We obtain then the first long exact sequence. Proposition 6.5 implies the second long exact sequence. 


\section{References}

[1] M. Auslander, Isolated singularities and almost split sequences, In Representation Theory II, Lecture Notes in Mathematics, No. 1178 (Springer-Verlag, 1986), 194-242.

[2] L. Bican, R. El Bashir, E. Enochs, All modules have flat covers, Bull. London Math. Soc. (4) 33 (2001), 385-390.

[3] H. Cartan, S. Eilenberg, Homological algebra (Princeton Univ. Press, 1956).

[4] R. R. Colby, Rings which have flat injective modules, J. Algebra 35 (1975), 239-252.

[5] G. A. Garkusha, FP-injective and weakly quasi-Frobenius rings, Zap. Nauchn. Sem. S.-Peterburg. Otd. Mat. Inst. Steklov. (POMI) 265 (1999), 110-129. (Russian). English transl. in J. Math. Sci. (3) 112 (2002) (also available at www.pdmi.ras.ru/znsl).

[6] G. A. Garkusha, Grothendieck categories, Algebra i Analiz (2) 13 (2001), 1-68. (Russian). English transl. in St. Petersburg Math. J. (2) 13 (2002).

[7] G. A. Garkusha, A note on almost regular group rings, Zap. Nauchn. Sem. S.-Peterburg. Otd. Mat. Inst. Steklov. (POMI) 281 (2001), 128-132. (Russian). (also available at www.pdmi.ras.ru/znsl).

[8] G. A. Garkusha, A. I. Generalov, Duality for categories of finitely presented modules, Algebra i Analiz (6) 11 (1999), 139-152. (Russian). English transl. in St. Petersburg Math. J. (6) 11 (2000).

[9] G. A. Garkusha, A. I. Generalov, Grothendieck categories as quotient categories of (R mod, Ab), Fund. $i$ Prikl. Mat. (4) 7 (2001), 983-992. (Russian). (also available at www.math.msu.su/ fpm/rus/k01/k014/k01402h.htm)

[10] S. I. Gelfand, Yu. I. Manin, Methods of homological algebra. I (Nauka, 1988). (Russian).

[11] A. I. Generalov, Relative homological algebra in preabelian categories, I: Derived categories, Algebra $i$ Analiz (1) 4 (1992), 98-119. (Russian). English transl. in St. Petersburg Math. J. (1) 4 (1993).

[12] L. Gruson, C. U. Jensen, Dimensions cohomologiques reliées aux foncteurs $\lim ^{(i)}$, In Paul Dubreil and Marie-Paule Malliavin Algebra Seminar, Lecture Notes in Mathematics, No. 867 (Springer-Verlag, 1981), 234-294.

[13] I. Herzog, The Ziegler spectrum of a locally coherent Grothendieck category, Proc. London Math. Soc. (3) $\mathbf{7 4}$ (1997), 503-558.

[14] S. Jain, Flat and FP-injectivity, Proc. Amer. Math. Soc. (2) 41 (1973), 437-442.

[15] C. U. Jensen, Les foncteurs dérivés de $\varliminf^{(i)}$ et leurs applications en théorie des modules, Lecture Notes in Mathematics, No. 254 (Springer-Verlag, 1972).

[16] H. Krause, The spectrum of a locally coherent category, J. Pure Appl. Algebra 114 (1997), 259-271.

[17] H. Krause, Functors on locally finitely presented additive categories, Colloq. Math. 75 (1998), 105-132.

[18] H. Krause, The spectrum of a module category, Habilitationsschrift. (Univ. Bielefeld, 1998), Mem. Amer. Math. Soc. 149 (2001), No. 707.

[19] M. Prest, Ph. Rothmaler, M. Ziegler, Absolutely pure and flat modules and "indiscrete" rings, J. Algebra 174 (1995), 349-372.

[20] D. Quillen, Higher algebraic K-theory. I, In Algebraic K-theory I, Lecture Notes in Mathematics, No. 341 (Springer-Verlag, 1973), 85-147.

[21] Ph. Rothmaler, When are pure-injective envelopes of flat modules flat, Comm. Algebra (6) 30 (2002), 3077-3085.

[22] E. G. Sklyarenko, Relative homological algebra in categories of modules, Uspekhi Mat. Nauk (3) 33 (1978), 85-120. (Russian). English transl. in Russian Math. Surveys (3) 33 (1978).

[23] E. G. Sklyarenko, Pure and finitely presented modules, duality homomorphisms and the coherence property of a ring, Mat. Sb. (2) 105 (1978), 192-206. (Russian). Engl. transl. in Math. USSR-Sb. 34 (1978).

[24] B. Stenström, Coherent rings and FP-injective modules, J. London Math. Soc. (2) 2 (1970), 323-329.

[25] B. Stenström, Rings of quotients (Springer-Verlag, 1975).

[26] R. G. Swan, Higher algebraic K-theory, Proc. Symposia in Pure Math. 58.1 (1995), 247-293.

[27] J.-L. Verdier, Catégories dérivées, In Séminaire de Géométrie Algébrique Du Bois-Marie (SGA $4 \frac{1}{2}$ ), Lecture Notes in Mathematics, No. 569 (Springer-Verlag, 1977), 262-311.

[28] C. A. Weibel, An introduction to homological algebra (Cambridge University Press, 1995).

[29] B. Zimmermann-Huisgen, W. Zimmermann, On the sparsity of representations of rings of pure global dimension zero, Trans. Amer. Math. Soc. 320 (1990), 695-711.

St. Petersburg State University, Department of Mathematics and Mechanics, Universitetsky PR. 28, 198504, Stary Peterhof, Russia

E-mail address: ggarkusha@mail.ru 